\title{
Contraste teórico de ratios en residencias de mayores desde la asociación entre el índice Barthel y el Baremo de Valoración de la Dependencia (BVD)
}

\author{
Alejandro Gómez Ordoki \\ ago2804@gmail.com
}

Geriatriako laguntzaileen ratioak esleitzeko Estatuko legedia desberdinen arteko bereizketa errealitate ukaezina da. Beste ondorio batzuen artean, egoera horrek egoitza-artarako baliabideen esleipen-eredu erabat heterogeneoak sortarazten ditu. Ratioak ez dira baliokide gertatzen, eta ez dute aurkezten baremo edo eskala berarekin neurtutako homogeneoak diren profilik; eta are gehiago, ezin diete eutsi haien ondorio zuzen diren arta-prezioen definizioei. Sektorearen ezaugarri diren alde nabarmen horiei Mendekotasunaren Balorazio Baremoak aurreikusitako mendetasunmaila bakoitzean inplizituki dauden profilen kopuru handia gehitzen badiegu, emaitzak sakabanaketa areagotzen $\mathrm{du}$, enpirikoki frogatzen saiatuko garen bezala. Azterlan honen helburua desberdinen arteko baliokidetasun-faktore potentzial bat mugatzea da, Estatuko egungo ratioen nahikotasuna eztabaidatu nahi ez badu ere, gutxienez, ratio horiek erreferentzia beraren eta neurketa-eredu beraren arabera kontrastatzea bilatzen duena.

\section{GAKO-HITZAK:}

Barthel Indizea, mendekotasunaren indize haztatua, Moretag, Denadat, gerokultorea, adineko pertsonentzako egoitzak, mendekotasuna.
La dispersión entre las diferentes normativas del Estado para la asignación de ratios de auxiliar de geriatría es una realidad innegable. Entre otras consecuencias, tal escenario origina modelos de asignación de recursos para la atención residencial de personas mayores absolutamente heterogéneos. $\mathrm{Ni}$ las ratios son equivalentes, ni representan a perfiles más o menos homogéneos medidos conforme a un mismo baremo o escala ni, en menor medida aún, soportan la definición de unos precios de atención que sean consecuencia directa de estas. Si a este conjunto de evidentes diferencias que caracteriza al sector le añadimos la enorme amplitud de perfiles implícitos en cada grado de dependencia contemplado por el Baremo de Valoración de la Dependencia, el resultado acrecienta la dispersión, como empíricamente trataremos de demostrar. El presente estudio intenta delimitar un factor de potencial equivalencia entre distintos que, si bien no pretende discutir la suficiencia de las actuales ratios del Estado sí, al menos busca contrastarlas conforme a una misma referencia y a idéntico patrón de medición.

\section{Palabras ClaVe:}

Índice Barthel, índice ponderado de dependencia, Moretag, Denadat, ratio de gerocultor, residencias para personas mayores, dependencia. 


\section{El paradigma actual en la asignación de ratios de auxiliar de geriatría}

La estimación de tiempos de auxiliar de geriatría ha constituido una constante preocupación no planteada de modo consensuado y transparente hasta la fecha. Con el propósito de objetivar su necesidad en términos de horas de atención, y bajo el mecenazgo de Lares Euskadi, nace Moretag (Modelo Referencial de Tiempos de Auxiliar de Geriatría), un modelo teórico de asignación de ratios estandarizadas de gerocultor/a en base a la experiencia práctica en siete geriátricos de Gipuzkoa y Bizkaia. El proyecto desarrollado busca que todos los agentes sociales que interactúan en la prestación de servicios residenciales para personas mayores se expresen con un lenguaje común y propio. Si bien las diferentes Administraciones Públicas han ido adaptando progresivamente los modelos de atención a la realidad cambiante del sector, se entiende que las organizaciones que lo conforman han alcanzado la suficiente madurez profesional como para proponer nuevos diseños basados en sus propios conocimientos empíricos. Con la participación de entidades de distintos territorios forales, además de pretender la definición de un marco común de actuación, se contextualiza la prestación del servicio en centros geográfica y culturalmente homogéneos.

Probablemente Moretag sea la única herramienta de ámbito estatal que mide la necesidad de auxiliar de geriatría en función del mix de dependencia. En ese cometido describe perfiles de atención derivados del índice combinado Barthel-CIF, gráficamente representado en el eje de abscisas por una combinación de los módulos del índice Barthel y del Baremo de Valoración de la Dependencia (alimentación, usar el retrete, deposición/ micción, higiene personal, acicalamiento, vestirse, transferencias, desplazamiento y mantenimiento de la salud) y, en el eje de ordenadas, por los grados de dependencia propuestos por la Clasificación Internacional del Funcionamiento, de la Discapacidad y de la Salud de la Organización Mundial de la Salud (CIF/OMS): autónomo -independiente-, leve, moderado, grave y completo. El referido índice Barthel-CIF está compuesto por aquellos módulos que, tanto en el Barthel -versión Shah-como en el Baremo de Valoración de la Dependencia (BVD), presentan, al menos teóricamente, un mínimo de ayuda de tercera persona para la realización de las actividades básicas de la vida diaria (tareas de auxiliar de geriatría). En este sentido, no solo se constituye en un método reproducible para la estimación de ratios; su mayor valor añadido, la base sobre la que se sustenta su desarrollo, es la definición de un marco referencial de tareas y tiempos unitarios implícitos en la atención directa (catálogo de servicios).

El despliegue normativo derivado de la transferencia de determinadas competencias a las comunidades autónomas ha desembocada en soluciones divergentes. No hay apenas simetría entre el precio y el perfil de dependencia y, en menor medida si cabe, entre la cualidad y la cantidad de ratios exigidas. El mapa autonómico de precios y ratios se caracteriza por la dispersión, no solo conceptual de lo que entendemos por categorías de dependencia sino, sobre todo, por las evidentes diferencias cuantitativas en los valores asignados a ambas variables (precio y ratio). Si, además, los precios no mantienen una relación directa con el coste de personal - a su vez, vinculados a las ratios de atención-, la idea de homogeneizar servicios esenciales que dan respuesta a derechos subjetivos de la ciudadanía empieza a ser necesaria por urgente y justa. Un horizonte aparentemente tan disperso e inconexo como el descrito no parece respetar el principio de solidaridad interterritorial promulgado por una Constitución garante del "equilibrio económico, adecuado y justo, entre las diversas partes del territorio español” (artículo 138).

Moretag define matrices de tiempos directos (diurnos y nocturnos) y valores de tiempos indirectos que, distribuidos conforme a los posibles binomios módulo-dependencia de la valoración Barthel-CIF, posibilitan la obtención de ratios de auxiliar de geriatría. Sin embargo, la traslación directa de dichas ratios a contextos asistenciales diferentes a los propios del estudio no sería aconsejable por el efecto esperado de dos variables principales: la ratio de atención y la jornada laboral anual. No debemos obviar que dos personas con una historia de vida similar, contribuyentes al Estado de Bienestar en medida y aportación semejantes, podrían ser perceptoras de diferentes calidades de servicio por mor de su padrón. En apenas seis kilómetros, dos localidades como Leintz Gatzaga (Gipuzkoa) y Landa (Araba), presentan dos marcos asistenciales absolutamente incomparables en una misma comunidad autónoma (Euskadi); entre Fuentes de León (Badajoz-Extremadura) y Cañaveral de León (Huelva-Andalucía), cada kilómetro de distancia - nueve en total- equivaldría a incrementar respectivamente la ratio extremeña de auxiliar de geriatría en un $16,6 \%$ para grados o y en un promedio del 7,2 \% para grados I y II/III, hasta asimilarlas a las ratios andaluzas. En consecuencia, determinar un buen "traductor" para transponer ratios entre diferentes normativas parece constituirse en una cuestión ineludible. Con ese ánimo se presenta un estudio que aspira a ofrecerse como una herramienta que, además, permitiría gestionar las necesidades de auxiliar de una manera equilibrada, eficiente y conforme a un determinado conjunto de tareas, directas e indirectas, inherentes a la prestación del servicio cualquiera que sea la forma que la misma adopte (modelos tradicionales de corte hostelero, modelos de atención gerontológica centrada en la persona -AGCP- o métodos lean).

Como en Moretag, trataremos de analizar la necesidad de ayuda de tercera desde una perspectiva científica. Cada output residencial (un día de atención o estancia) es normalmente distinto del anterior y, aun cuando aparentemente fueran iguales, cada 


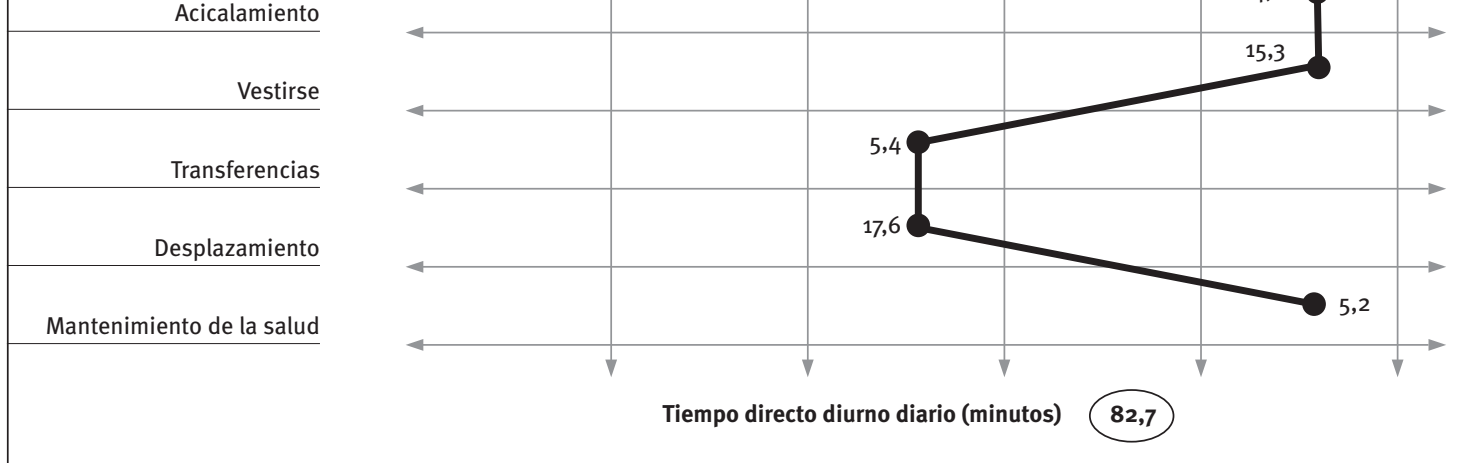

Fuente: Elaboración propia.

uno de ellos presentaría una composición diferente (Gómez, 2014). Si Moretag trata la aleatoriedad intrínseca en la prestación del servicio - distintos perfiles de atención para personas diferentes y/o variabilidad diaria en la atención de una misma persona- para proponer tiempos medios de atención estadísticamente significativos y con un alto grado de verosimilitud (desviaciones agregadas del $1,18 \%$ entre el modelo y la realidad y del o,15\% entre aquel y la agrupación por perfiles estándar o isogrupos), la evolución del modelo debería llevarnos a tratar también la aleatoriedad de perfiles (un total de 1.953.125 valoraciones Barthel-CIF diferentes) y su asociación con los diferentes grupos oficiales de dependencia (grados o, I, II y III del BVD). Ninguna Administración Pública se plantearía regular semejante volumen de posibles perfiles de atención, razón que conduce a la inevitable definición de "pasarelas" entre la puntuación total del índice Barthel y los grados de dependencia del BVD en términos de desviación mínima agregada para una determinada capacidad residencial (diferencia entre el tiempo total de auxiliar obtenida desde Moretag para cada perfil personalizado y el tiempo total propuesto desde la referida "pasarela"). En todo caso, si bien la adaptación teórica de los tiempos estandarizados de auxiliar propuestos por Moretag es una de las principales utilidades del modelo propuesto, deberíamos profundizar en la investigación de sus potenciales aplicaciones para la identificación de nuevos e innovadores usos de la herramienta (ver Figura 2).
GRAVEDAD

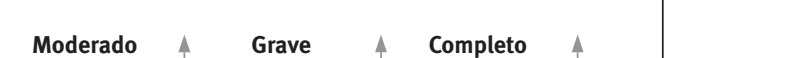
comparativa estatal de ratios de atención. Partiendo del modelo original, se plantea una metodología para la transposición de ratios entre las diferentes normativas del Estado aprovechando el potencial sinérgico del índice Barthel para agrupar todo el abanico posible de combinaciones de perfiles de dependencia desde la reducción de los mismos a los grados contemplados por el BVD (ideas de isogrupo y de factor de equivalencia entre normativas) y poder describir, en consecuencia, un mapa estatal de ratios conforme a un sistema homogéneo de cálculo. Esta voluntad por transformar Moretag en una herramienta transversal se basa en cuatro razones que justifican su necesaria evolución hacia nuevos ámbitos de aplicación:

- Progresivo envejecimiento de la población, aumento de la esperanza de vida y creciente demanda de soluciones residenciales.

- Grado de dependencia que concede derechos subjetivos, pero no detalla el nivel de ayuda para las actividades básicas de la vida diaria en un entorno residencial.

- Análisis de la composición del coste por plaza: el gasto en auxiliar de geriatría se sitúa en el intervalo del 40\%-50\% del coste total.

- Plantillas de auxiliares correlacionadas con el nivel de dependencia, equitativas y adaptables a cualquier modelo asistencial. 


\section{1. Índice Barthel: una sinergia irrenunciable}

No hay argumento racional que contradiga la teórica relación directa entre el precio y el coste de personal en los servicios residenciales para personas mayores. Comparativamente, la sensibilidad del precio a otros costes (funcionamiento y amortización) es casi residual; más aún, el personal de atención indirecta (básicamente servicios generales y de mantenimiento) tampoco condicionaría su valor. En otras palabras, el precio, ceteris paribus, es función directa de la ratio de atención directa. Y, dentro de los colectivos profesionales que conforman dicha atención, el trabajo desempeñado por las auxiliares de geriatría, por fundamental e insustituible, demanda un análisis específico.

La mayoría de las pautas emitidas para procurar el cumplimiento de los derechos inalienables de las personas mayores atendidas en geriátricos son ejecutadas por auxiliares de geriatría. Derechos, por otra parte, directamente vinculados con las siete dimensiones de la calidad de vida (De la Cámara, 2003) y con las nuevas tendencias en el enfoque de la atención derivadas de la psicología humanista (atención centrada en la persona). El trabajo de auxiliar, directa o indirectamente, está presente en todos los procesos de atención y, en consecuencia, es el punto sobre el que gira toda actividad en un geriátrico. A su vez, es la figura profesional que más efectiva hace la misión, visión y valores para el entorno sociofamiliar del residente (Gómez, 2018). Podríamos afirmar, con total seguridad, que es el recurso principal para garantizar una adecuada prestación del servicio.

No deberíamos proponer hipotéticas adecuaciones de Moretag como modelo de referencia para la asignación de plantillas de auxiliar de geriatría si previamente no hemos definido cómo y con qué frecuencia se mide la dependencia. En la publicación del Imserso "Discapacidad/deficiencia, unificación de criterios de valoración y clasificación" (Querejeta, 2004), se identifican hasta 21 tipos de escalas diferentes para determinar niveles de dependencia en función de la necesidad de ayuda, técnica o personal, para la realización de una determinada actividad de la vida diaria (AVD). En cualquier caso, el índice Barthel es el único baremo de presencia prácticamente garantizada dada su incorporación a la mayoría de los softwares generalistas de gestión residencial implantados en el conjunto del Estado. Esta previsible valoración de la dependencia conforme a una escala compartida y a una frecuencia mínima de administración (normalmente anual) avala su uso como estándar de medida, en un proceso sinérgico que no debería ser soslayado. El problema radica en que, si bien se constata la programación de valoraciones Barthel en la mayoría de los centros residenciales, estas no otorgan carácter de oficialidad a la dependencia y, en consecuencia, debe identificarse algún tipo de correspondencia con el baremo más extendido en el Estado para determinar perfiles de atención: el
Baremo para la Valoración de la Dependencia (BVD). La "pasarela” Barthel-BVD facilitará la adecuación de Moretag a cada normativa existente en el Estado desde factores de equivalencia para poder describir, asimismo, comparativas intercomunitarias basadas en distribuciones por isogrupos (grupos homogéneos con los que clasificar diferentes perfiles de dependencia) que, a su vez, asociaremos con cada uno de los grados contemplados por el BVD.

\subsection{Moretag como herramienta para la "homogeneización" de ratios}

Como ya se ha apuntado, Moretag es una herramienta que dimensiona, para un determinado contexto asistencial (centros integrantes del estudio), las plantillas de gerocultor/a en función de la composición o mix del colectivo a atender. Nuestra metodología no debería romper bajo ningún pretexto el supuesto equilibro entre el número de gerocultoras/es y el nivel de dependencia. Este y no otro debería ser el sentido de la evolución: determinar un sistema reproducible capaz de simular resultados verosímiles para todas las configuraciones imaginables de perfiles promedios en un centro residencial. Sin embargo, fotografiar la realidad cada cierto tiempo como propone www.moretag.org, si bien permite deducir el sentido de la evolución, no facilita argumentos para discriminar las causas de las tendencias y los puntos de inflexión que marcan los cambios. En otras palabras, más que fotografías necesitamos fotogramas que muestren el movimiento o, lo que es lo mismo, la trazabilidad de la información. Porque el cambio, por ejemplo, podría ser consecuencia de sesgos malintencionados (valoraciones Barthel viciadas hacia la dependencia grave o completa para justificar supuestas necesidades extras de personal) que la referida herramienta web no detecta. En consecuencia, la evolución del modelo debería desplegar una batería de indicadores y gráficos que nos guiarán en la interpretación de una realidad asistencial cambiante, que debería ser analizada, además, en ciclos temporales para poder proyectar escenarios de futuro al objeto de cumplir las exigencias venideras o, en su defecto, adaptar nuestras organizaciones para minimizar la falta de atención por insuficiencia de recursos necesarios.

Sin valoraciones Barthel no conoceríamos la estructura de la dependencia y, por consiguiente, no cabría la asignación de tiempos de gerocultor/a. En consecuencia, asegurar una gestión rigurosa y eficaz de las valoraciones programadas se antoja imprescindible para toda organización. El esquema de la Figura 2 conecta dos planos simétricos, Moretag y su evolución, para trasmitir una idea de continuidad entre el primero y el segundo que permita examinar los datos desde una perspectiva dinámica. Cuando una herramienta es estática y efímera (no guarda información histórica) no debería constituirse en un método adecuado para estimar tendencias, máxime en recursos con altísimos índices de rotación (se 
MORETAG: tiempos de gerocultor/a por perfiles de dependencia (prespectiva estática)

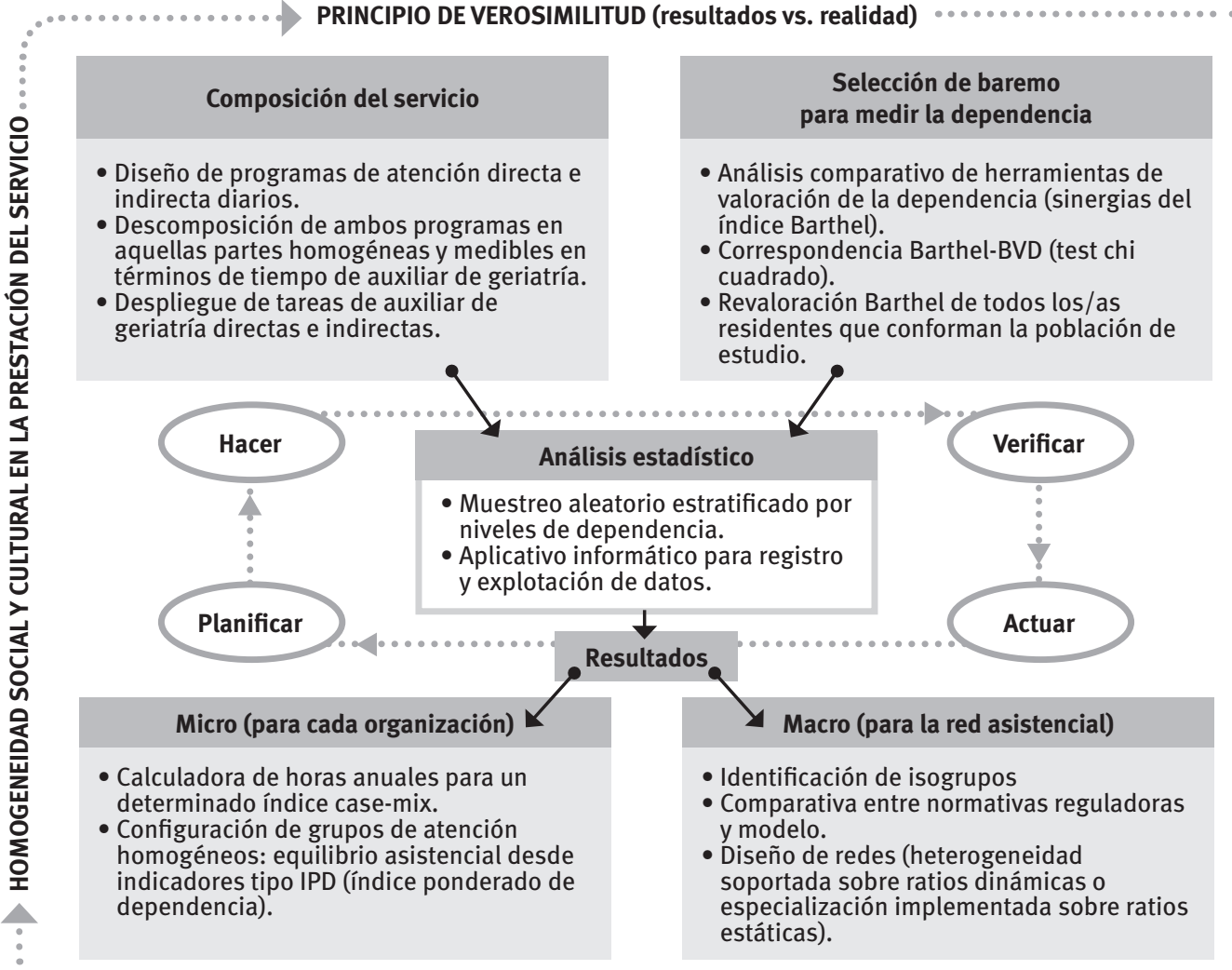

Despliegue de tareas de auxiliar de

.

cuadrado).

Revaloración Barthel de todos los/as residentes que conforman la población de

ADAPTABILIDAD A OTRAS REALIDADES ASISTENCIALES

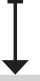

Evolutivo de necesidades de gerocultor/a y proyección de escenarios (perspectiva dinámica)

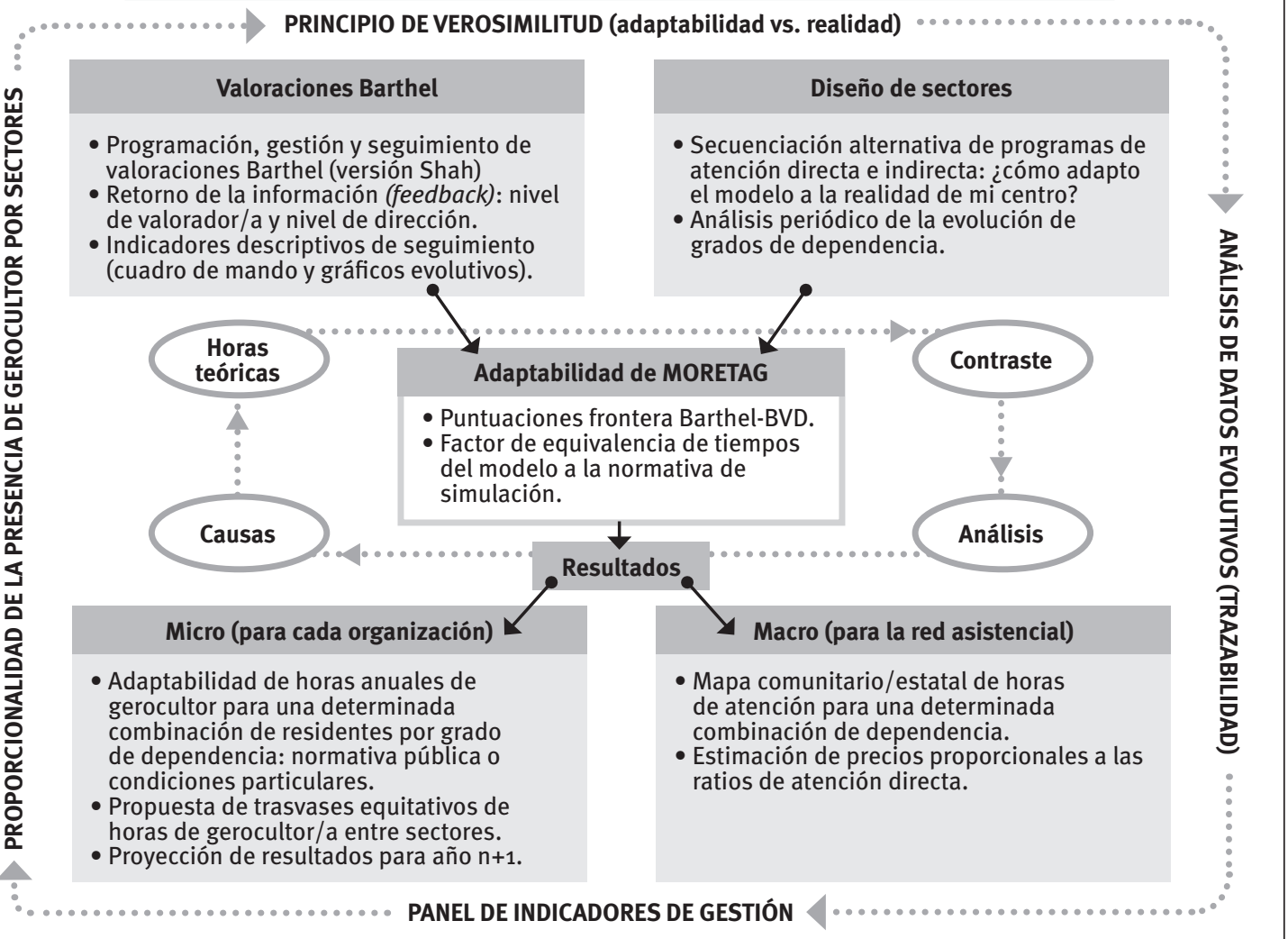

Fuente: Elaboración propia. 
despreciarían un número considerable de secuencias baja-alta de usuarios si solo contemplamos las valoraciones de los residentes de alta a una determinada fecha y no durante todo un periodo).

Moretag plantea tiempos estandarizados a resultas de las mediciones en un determinado contexto asistencial que, a su vez, ha implementado sus modelos de atención de acuerdo con las posibilidades propias que sus respectivas administraciones públicas han determinado (por ejemplo, el Decreto Foral 38/2007, por el que se determinan las condiciones necesarias para la concertación de servicios residenciales para personas mayores dependientes en Gipuzkoa). Por ello, como cabría esperar, los estándares de Moretag no son exportables a otras comunidades porque no comparten la misma normativa. Sin embargo, si aceptáramos que el catálogo de servicios es transversal al conjunto de autonomías del Estado -no hay razones objetivas para pensar que el conjunto de tareas directas e indirectas es consustancial a cada autonomía porque, entre otras evidencias, todos los convenios colectivos de aplicación en el Estado relacionan un marco similar de funciones para las auxiliares de geriatríapodríamos definir un factor de equivalencia o de adaptabilidad para Moretag cuyos efectos y validez ya han sido tratados en Gómez Ordoki, 2018).

\subsection{Metodología}

Moretag ha sido un proyecto desarrollado conforme a una metodología muy concreta. La obtención de la matriz de tiempos directos de gerocultor/a por nivel de dependencia y de la tabla de tiempos indirectos ha supuesto la culminación de un proceso que debería continuar en la generación de un "transponedor" de los tiempos de auxiliar $-y$, consecuentemente, de las horas anuales de atención y de las plantillas de personal necesariasa normativas diferentes de las que delimitan el marco de estudio. El resultado de la transposición no es, literalmente, la obtención de unas nuevas ratios sustitutorias para la normativa comparada aunque cabría dicha posibilidad teórica, como más adelante trataremos. El contraste se revela como un cotejo homogéneo, para un determinado mix de dependencia, entre las horas/año establecidas según normativa y las horas derivadas desde la aplicación de Moretag adaptado según el factor de equivalencia $p$. El método de contraste de ratios desarrollado ("DENAdat-herramienta para el dimensionamiento de plantillas de gerocultor/a") se muestra como un modelo de medición que produce retornos de información a dos niveles: agregado o de la residencia en su conjunto - de interés para los profesionales responsables de la valoración Barthel-, y sectorizado o de cada unidad de atención en particular - diseñado para responder de las necesidades propias de los órganos de dirección del geriátrico-. Metodológicamente, el modelo no es sino un conjunto de bases de datos que registran y guardan el histórico de valoraciones Barthel por unidades de atención que, encuadradas en los respectivos diseños particularizados de los programas diarios de atención directa e indirecta, proporcionan información fehaciente para la toma de decisiones, tanto desde una perspectiva integral (centro particular y/o conjunto de centros de una misma organización o unidad político administrativa) como desde cada una de las unidades de atención o sectores de cada centro residencial.

El método de contraste entre normativas busca cumplir un doble objetivo cualitativo y cuantitativo: por un lado, reducir todas las posibles combinaciones del binomio actividades de la vida diaria-dependencia a un número limitado y estadísticamente representativo de grupos homogéneos (isogrupo) y, por otro, analizar, en clave de benchmarking, las necesidades de atención en función de perfiles estandarizados de dependencia. Aunque el modelo no viene a solucionar la innegable dispersión estatal que, por otra parte, no es sino

Figura 3. Diseño de un modelo para la proyección de resultados a otros contextos

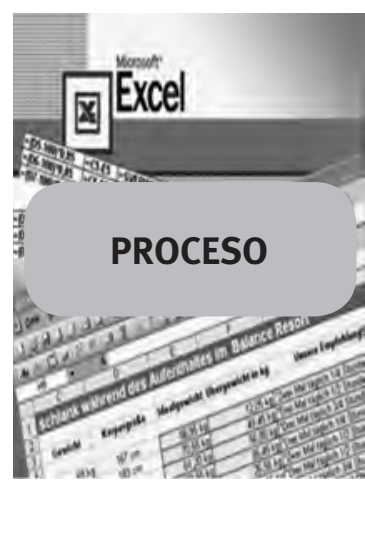

APLICACIONES DEL MODELO

Registro y gestión cíclica de valoraciones Barthel-Shah.

Simulación de resultados adaptados a nuevas ratios de atención (normativas comunitarias, acuerdos bilaterales, etc.) y adaptación de catálogos de tareas alternativos.

Resultados particularizados por unidades de atención y proyección de escenarios futuros.

Fuente: Elaboración propia. 
un reflejo del contexto socioeconómico de cada autonomía (salarios e índice de coste de la vida) y del ideario asistencial defendido por cada ejecutivo autonómico, sí podría llegar a ubicar cada centro en su entorno inmediato (organización e, incluso, autonomía o territorio, este último en el caso de la Comunidad Autónoma del País Vasco) y/o simular, al menos desde una perspectiva teórica y con los datos disponibles, una comparativa estandarizada entre distintas normativas.

Como posibilidad de futuro, supuesta una correlación consistente entre las diferentes categorías profesionales de atención directa y estandarizados los módulos de coste diferentes a los de personal directo, el modelo podría ser el embrión metodológico a partir del cual desarrollar un método para homogeneizar el cálculo del precio, habida cuenta del esperado paralelismo entre su valor y las variaciones en el coste de personal por efecto de cambios en el valor de las ratios. Dada la inalcanzable capacidad de contar con datos fidedignos para las distintas normativas autonómicas, solo desde la imaginación podremos diseñar modelos de comparación basados en la lógica esperada para la relación directa entre precio y ratio. Por ello, la búsqueda de concordancias con otros modelos de estimación de tiempos de gerocultor/a - si los hubiera- es ciertamente dificultosa. En un escenario semejante, la “transposición” ideada quizá pudiera aplicarse como regla de paridad para contrastar ratios con criterios de homogeneidad. Por el momento, no se adivinan caminos alternativos

\subsection{Impactos positivos de una hipotética homologación de ratios}

De homologarse las ratios, los impactos positivos que se producirían serían los siguientes:

\section{- Reivindicación del/la gerocultor/a como} figura profesional fundamental. El éxito de las organizaciones residenciales dependerá del apoyo sincero e incondicional a la labor desempeñada por el personal auxiliar. La observación de la realidad demuestra que la mayoría de las pautas emitidas para procurar el cumplimiento de las siete dimensiones de un modelo de calidad de vida, como el modelo de Schalock-Verdugo (Imserso, 2011), son ejecutadas por auxiliares de geriatría porque, directa e indirectamente, están presentes en todos los procesos de atención y en esa interacción se constituyen en el punto nuclear sobre el que gira la atención en un geriátrico.

- Innovación. El verdadero potencial de Moretag y las aplicaciones derivadas está aún por descubrir. Su límite está en la capacidad para imaginar nuevos usos y en esta búsqueda no deberíamos delimitar fronteras que constriñan nuestro avance. A futuro se podrían dar posibilidades suficientes para obtener inferencias desde la presumible correlación con otros profesionales de atención directa y, así, poder dar valor al precio, ceteris paribus, como función directa de la ratio de atención directa.

- Generación de empleo. Abordar las exigencias de una adecuada respuesta a la evolución experimentada por el perfil promedio en las dos últimas décadas (residentes con edades medias muy avanzadas y pluripatologías físicas y/o psíquicas) incrementaría las ratios previstas por normativas antiguas e insensibles a la composición mix del servicio. Es de prever que se reclamen mayores plantillas de personal en un mercado teóricamente estable por el progresivo deterioro psicofísico del residente tipo y el aumento de la esperanza media de vida (según la revista digital Dependencia.info, las previsiones del Informe DBK apuntan hacia un crecimiento sostenido del sector residencial en el Estado).

- Tecnificación del cálculo de recursos necesarios. Impulsar nuestra credibilidad como organizaciones profesionalizadas desde el uso de herramientas técnicas al servicio de logros sociales. Abandonar la asignación de ratios por ciencia infusa para justificar la necesidad de contar con ellos desde métodos basados en el rigor científico debería ser un norte innegociable. Debemos empezar a dejar de ser beneficencia para paulatinamente regenerarnos en "beneficiencia" sin perder ninguno de nuestros principios y, desde luego, nunca a cualquier precio.

- Homogeneización de precios y costes. La dispersión del mapa estatal de precios aconseja modular su cálculo conforme a un mismo patrón, en relación directa con la ratio de atención directa que, a su vez, debería tender a una progresiva reducción de las actuales diferencias intercomunitarias. Desde enfoques compartidos ayudaríamos a homogeneizar los servicios o, al menos, a homologar el cálculo del precio público y "remendar" un tejido residencial ciertamente descompensado.

\section{Comparativa estatal: ratios en normativa versus ratios según factor de equivalencia de Moretag}

\subsection{Relación entre tiempo de atención y puntuación total del índice Barthel}

Como se ha comentado, el índice Barthel es una escala de uso común que se administra al menos con frecuencia anual, y que soporta la asignación de tiempos de auxiliar de geriatría en función del nivel de ayuda de tercera persona para la realización de las actividades de la vida diaria (AVD). Este índice, sin embargo, no determina oficialmente el grado de dependencia para personas mayores en residencias en ninguna de las comunidades del Estado, a excepción de la Comunidad Valenciana. 
En consecuencia, es necesaria la traslación de los tiempos unitarios por usuario y día de Moretag a los grados de dependencia del BVD que, por otra parte, no por oficial es la referencia generalmente compartida por las diferentes normativas del Estado. De las diecinueve normas revisadas (dieciséis comunidades autónomas y tres territorios históricos -Araba, Gipuzkoa y Bizkaia-, exceptuadas las ciudades autónomas de Ceuta y Melilla), tan solo ocho contemplan el BVD como baremo para discriminar perfiles de dependencia; para el resto de normativas (dos con escala diferente al Barthel y nueve sin escala definida), la oficialidad del BVD aconsejaría su uso como prescriptor de dicho perfil.

Moretag estima necesidades de gerocultor/a desde una matriz de tiempos unitarios que asigna valores a cada combinación de módulo (filas) y nivel de dependencia (columnas). Cada índice Barthel, también una matriz de módulos de atención y capacidad para la realización de AVD, se reconvierte en un perfil Barthel-CIF al que se adjudican los correspondientes tiempos unitarios. El resultado total es la suma del tiempo unitario estandarizado de cada binomio módulo-nivel de dependencia (tal y como se podía apreciar en la Figura 1).

Si el valor del tiempo unitario por punto es propio y particular de cada binomio módulo-nivel de dependencia, no puede concluirse que el tiempo total de atención es función directa de la puntuación total Barthel. De igual manera, como demuestra Moretag, el tiempo máximo de gerocultor/a no se corresponde con el perfil que adopta el nivel de dependencia máximo (categoría “completo") para todos y cada uno de los módulos Barthel-CIF. En otras palabras, es la composición del perfil de atención la que determina el tiempo y no la puntuación total. En consecuencia, no podrían identificarse puntuaciones que delimitaran fronteras absolutamente infranqueables entre los diferentes grados de dependencia. La solución pasaría por la fijación de puntuaciones totales del índice Barthel que minimizaran la desviación promedio para cada grado del BVD, asumiendo que, dada la aleatoriedad de perfiles, la presencia de cada perfil en cada grado debería ser teóricamente equiprobable. A priori, no podríamos defender que determinados perfiles son más frecuentes que otros - más probablesporque, en principio, cada nivel de dependencia para cada módulo del índice Barthel -independiente, ligero, moderado, grave y completo- es igualmente valorable. Empíricamente, sin embargo, nos encontrarnos con una realidad que describe cierta concentración de perfiles de atención. Así, esta nos sugiere que la función de probabilidad nunca se trazaría como una línea recta paralela al eje de abscisas - absoluta equiprobabilidad-sino como un polígono de frecuencias con una mayor concentración de valores, por término medio, para las categorías “independiente" y "completo" que para el resto que, por su parte, sí podríamos interpretar como equiprobables en su conjunto, al menos para la población de estudio en Moretag (Gómez, 2018). Esta deducción a posteriori no debería, en ningún caso, condicionar las premisas teóricas.

Para demostrar que "a menor puntuación Barthel, mayor tiempo de auxiliar” es una hipótesis de trabajo fallida, construiremos dos bases de datos que se constituyan, a su vez, en muestras aleatorias con suficiente tamaño para ser estadísticamente significativas (por un lado, $\mathrm{N}$ $=300$ o tamaño correspondiente a la residencia de mayor capacidad en Moretag $\mathrm{y}$, por otro, $\mathrm{N}=$ 5.000 o muestra para dar cabida a un número suficiente de diferentes perfiles equiprobables) y sobre las que simularemos diferentes resultados para comprobar el incumplimiento de la hipótesis planteada. En aplicación de lo que establecen las leyes de la inferencia estadística, probaremos tal incumplimiento en 30 simulaciones diferentes. El resultado de esta prueba empírica se resume en la siguiente tabla:

Tabla 1. Diferente ponderación temporal de cada punto Barthel

\begin{tabular}{|l|c|c|c|c|c|}
\hline \multicolumn{5}{|c|}{ Puntuación índice Barthel } \\
\hline \multirow{2}{*}{ Módulo } & \multicolumn{5}{c|}{ Dependencia* } \\
\cline { 2 - 7 } & I & L & M & G & C \\
\hline Alimentación & 10 & 8 & 5 & 2 & 0 \\
\hline Ir al retrete & 10 & 8 & 5 & 2 & 0 \\
\hline Micción & 10 & 8 & 5 & 2 & 0 \\
\hline Baño & 5 & 4 & 3 & 1 & 0 \\
\hline Aseo & 5 & 4 & 3 & 1 & 0 \\
\hline Vestido & 10 & 8 & 5 & 2 & 0 \\
\hline Traslado & 15 & 12 & 8 & 3 & 0 \\
\hline Deposición & 10 & 8 & 5 & 2 & 0 \\
\hline Deambulación & 15 & 12 & 8 & 3 & 0 \\
\hline Escaleras & 10 & 8 & 5 & 2 & 0 \\
\hline
\end{tabular}

* $\mathrm{I}=$ Independiente, $\mathrm{L}=$ Ligero, $\mathrm{M}=$ Moderado, $\mathrm{G}=$ Grave,

$\mathrm{C}=$ Completo.

\begin{tabular}{|c|c|c|c|c|c|c|c|c|c|c|}
\hline \multicolumn{11}{|c|}{ Tiempos unitarios Barthel-CIF } \\
\hline \multirow{2}{*}{ Módulo } & \multicolumn{5}{|c|}{ Tiempos unitarios (minutos/día) } & \multicolumn{5}{|c|}{ Tiempo por punto } \\
\hline & $\mathbf{I}$ & $\mathbf{L}$ & M & G & C & I & $\mathbf{L}$ & $M$ & G & C \\
\hline Alimentación & 6,12 & 12,58 & 8,06 & 19,72 & 32,67 & 0,61 & 1,57 & 1,61 & 9,86 & \\
\hline Usar el retrete & 0,87 & 1,31 & 3,67 & 4,20 & 5,03 & 0,09 & 0,16 & 0,73 & 2,10 & \\
\hline Deposición/Micción & 0,90 & 0,98 & 2,05 & 2,20 & 3,71 & & & & & \\
\hline Higiene personal & 1,93 & 2,86 & 5,77 & 6,09 & 6,58 & 0,39 & 0,72 & 1,92 & 6,09 & \\
\hline Acicalamiento & 1,26 & 1,69 & 3,97 & 6,10 & 4,18 & 0,25 & 0,42 & 1,32 & 6,10 & \\
\hline Vestirse & 2,84 & 7,73 & 9,96 & 18,14 & 15,25 & 0,28 & 0,97 & 1,99 & 9,07 & \\
\hline Transferencias & 2,84 & 6,74 & 5,42 & 13,76 & 27,41 & 0,19 & 0,56 & 0,68 & 4,59 & \\
\hline Desplazamiento & 3,25 & 5,95 & 17,54 & 13,40 & 11,66 & & & & & \\
\hline Mantenimiento salud & 2,02 & 2,31 & 3,03 & 5,18 & 4,66 & & & & & \\
\hline
\end{tabular}

Traslación directa de puntos Barthel a puntos Barthel-CIF

Traslación combinada de puntos Barthel a puntos Barthel-CIF

Fuente: Elaboración propia. 
Tabla 2. Incumplimiento de hipótesis “a mayor puntuación Barthel, menor tiempo de auxiliar de geriatría” (relación indirecta)

\begin{tabular}{|c|c|c|c|c|c|c|c|c|}
\hline \multirow{3}{*}{ Muestra } & \multirow{3}{*}{ Estadístico } & \multirow{3}{*}{$\begin{array}{l}\text { Número de } \\
\text { comparaciones }\end{array}$} & \multicolumn{6}{|c|}{ Evidencias de contraste } \\
\hline & & & \multicolumn{2}{|c|}{$\begin{array}{l}\text { Puntuación: } \\
\text { igual o menor }{ }^{(1)} \\
\text { Tiempo: menor }\end{array}$} & \multicolumn{2}{|c|}{$\begin{array}{c}\text { Puntuación: } \\
\text { igual o mayor }{ }^{(2)} \\
\text { Tiempo: mayor }\end{array}$} & \multicolumn{2}{|c|}{ Totales } \\
\hline & & & № veces & $\begin{array}{c}\% \text { sobre } \\
\text { comparaciones }\end{array}$ & № veces & $\begin{array}{c}\% \text { sobre } \\
\text { comparaciones }\end{array}$ & № veces & $\begin{array}{c}\% \text { sobre } \\
\text { comparaciones }\end{array}$ \\
\hline \multirow{2}{*}{300} & Promedio & 13.193 & 1.589 & $12,1 \%$ & 1.672 & $12,7 \%$ & 3.261 & $24,8 \%$ \\
\hline & Desviación & 565 & 239 & $1,76 \%$ & 252 & $2,13 \%$ & 220 & $1,94 \%$ \\
\hline \multirow{2}{*}{5.000} & Promedio & 352.952 & 33.823 & $9,6 \%$ & 36.624 & $10,4 \%$ & 70.448 & $20,0 \%$ \\
\hline & Desviación & 6.660 & 4.505 & $1,25 \%$ & 5.172 & $1,47 \%$ & 5.605 & $1,56 \%$ \\
\hline
\end{tabular}

(1) Veces en las que para una determinada puntuación ocurre que en las simulaciones anteriores, a igualdad o inferioridad de puntos presentan menor tiempo.

(2) Veces en las que para una determinada puntuación ocurre que en las simulaciones anteriores, a igualdad o superioridad de puntos presentan mayor tiempo.

Fuente: Elaboración propia.

Los tiempos diarios no corroboran la hipótesis en entre un cuarto y un quinto de las simulaciones. Además, como cabría presumir, el incremento en el tamaño de la muestra tiende a reducir la incertidumbre en la medida que los resultados obtenidos se desvían en menor proporción sobre valor medio. En todo caso, un tamaño de muestra conformado por una base de datos con 300 observaciones aleatorias ofrecería resultados suficientemente fidedignos.

\subsection{Puntuaciones frontera Barthel-BVD}

La pasarela manejada por Moretag delimita el cambio de grado I a grado conjunto II/III en los 79 puntos del índice Barthel (como explicaron Alazne Izagirre y Juan José Calvo en diversos congresos en 2014). Esta frontera preliminar, basada en la prueba chi cuadrado a un nivel de confianza del 99,9\% para demostrar la existencia o no de independencia entre dos variables -en nuestro caso, entre el índice Barthel y el baremo para la valoración de la dependencia-, determinó el tamaño de cada estrato (autónomo, dependiente y gran dependiente, a su vez respectivamente equiparados a cada grado del BVD) en la obtención de una muestra aleatoria estratificada por afijación proporcional para el estudio del tiempo necesario de gerocultor/a en función del nivel de dependencia. Esta divisoria, en un principio válida para garantizar suficiente presencia de cada estrato de la población en la muestra aleatoria, podría no ser la que minimizara la desviación promedio entre los tiempos de Moretag y aquellos otros derivados de asociar la puntuación total Barthel con cada uno de los grados BVD. De hecho, la hipótesis nula - solo en un 0,1\% de los casos, el azar explicaría una relación inversa entre Barthel y BVD - se cumple para tal intervalo de puntuaciones Barthel que discriminar grados de dependencia desde este único valor (79 puntos) obviaría el contraste empírico necesario para validar la frontera. Por todo ello, ¿cómo proceder para que la correspondencia Barthel-BVD, conocida la posibilidad de tránsitos entre fronteras dado el incumplimiento parcial de la relación $\nabla$ Barthel $\rightarrow \Delta$ Tiempo, se produzca en términos de mínima desviación promedio?

Recordemos que para calcular el factor de equivalencia $(p)$ necesitamos conocer previamente el tamaño de cada estrato de dependencia (n.) para una determinada capacidad residencial o número total de personas atendidas (n). Como hemos apuntado, algunas normativas del Estado no detallan prescriptores de dependencia; otras, cuando lo hacen, no segregan perfiles de atención (grados I, II y III son elementos de un mismo conjunto cuando las diferencias de atención son más que evidentes). Si la normativa es tan avanzada que incluso diferencia entre grado II y grado III, ¿en base a qué soporte se solicita un cambio de valoración si se estima una mayor necesidad de auxiliar no derivada de circunstancias coyunturales? ¿Podría una residencia aplicar el BVD y que su dictamen adquiriera dimensión de oficialidad?

Sin la parametrización de cada estrato de dependencia no obtendremos el valor del coeficiente de adaptación y, consecuentemente, cualquier empeño en contrastar normativas desde una herramienta objetiva sería inviable. Aunque Moretag presumiblemente constituya la única referencia en el Estado para la asignación analítica de ratios desde una perspectiva científica empíricamente validada, no podremos observar su capacidad de transversalidad, su ánimo para ser proyectada a contextos asistenciales distintos al propio (Gipuzkoa y Bizkaia), si no se estandarizara la graduación de la dependencia desde escalas o baremos totalmente compartidos y sujetos a una valoración periódica programada. Indudablemente, el índice Barthel es el único capaz de respetar las exigencias de una pretendida transversalidad.

Moretag estima las horas de gerocultor/a como la suma anualizada del tiempo diario de atención estándar (diurno directo e indirecto y nocturno directo) para cada perfil de cada residente. Los tiempos medios diarios de cada binomio módulo 
Barthel-nivel de dependencia (atención directa) y de cada módulo de atención indirecta constituyen los únicos inputs necesarios para el cálculo del tiempo por residente y día. Algebraicamente expresado, podemos definir el tiempo necesario de auxiliar de geriatría para satisfacer las necesidades de una determinada tipología de residente como sigue (Gómez, 2015):

$$
t=\sum_{i=1}^{i=n} \sum_{j=0}^{j=4} c_{i} \cdot i_{j} \longrightarrow \begin{aligned}
& \text { el tiempo diario total de } \\
& \text { auxiliar } t \text { es igual a la } \\
& \text { suma de } i \text { componentes a } j \\
& \text { intensidades para cada uno } \\
& \text { de los } n \text { residentes. }
\end{aligned}
$$

En cualquier caso, el resultado de la expresión estaría sujeto a la aleatoriedad de los perfiles de atención. Normalmente cada perfil es un output distinto y propio y, en esa condición, el valor de $t$ se descompondrá en tantos sumandos como binomios modulo-dependencia presenten las personas residentes. El valor agregado, cambiante con cada alta, baja o modificación de las circunstancias de cada residente, se constituye en el umbral de tiempo anual alcanzable desde la asignación de ratios a cada grado oficial de dependencia según la pasarela Barthel_BVD. Huelga decir que el planteamiento del problema en términos de análisis matemático es de tal complejidad que su resolución se antoja prácticamente imposible. Por tanto, despejaremos la incógnita (puntuación frontera) desde métodos heurísticos mediante el uso de técnicas de tanteo y aproximación basadas en las opciones de cálculo que nos ofrecen herramientas como Excel.

En las dos bases de datos que conforman las muestras de estudio $(\mathrm{N}=300$ y $\mathrm{N}=5.000)$ simularemos aleatoriamente índices Barthel que, a su vez, concretarán perfiles Barthel-CIF sobre los que aplicar los tiempos estandarizados de Moretag para un día de atención. La equiprobabilidad en la adjudicación de niveles de dependencia a cada perfil simulado debe ser una máxima. Si bien la realidad refuta dicha necesidad, la muestra debería estar integrada por cuantos perfiles sean posibles. La estricta observancia de este requisito pasa por no subordinar a regla alguna la distribución de las categorías "independiente", "ligero", "moderado", "grave" y "completo". Como consecuencia, al contemplarse igualmente todos los niveles de dependencia, el índice ponderado de dependencia (en adelante IPD o, donde $n_{i}$ y $r_{i}$ son, respectivamente, los tamaños y ratios de cada estrato de dependencia) registra escasa oscilación porque no se producen sesgos por mayores frecuencias en la presencia de determinados niveles de dependencia y, en consecuencia, tiende a la centralidad estadística. Los estadísticos así obtenidos son reflejo de todo el abanico de posibles combinaciones e infieren parámetros de la población válidos para simular cualquier realidad. La concentración de perfiles determinará hasta qué punto divergen Moretag y normativa y cómo debe ser analizada la misma para minimizar sus efectos.

Estimaremos tiempos para los grados o, I y II/ III de manera que se complemente la desviación observada entre Moretag y la normativa que más se aproxima al modelo (Decreto 38/2007 de la Diputación Foral de Gipuzkoa), cifrada en torno al 6\%-7\% para una composición mix de dependencia con una evidente preponderancia de perfiles II y III (distribución tipo en Gipuzkoa a diciembre de 2018: $83 \%$ de grados II/III, $15 \%$ de grado I y $2 \%$ de grado o). Los tiempos unitarios que ajustan la referida desviación respetando, asimismo, los tiempos de Moretag para grados o y para simulaciones en número estadísticamente significativo de grados I y II/III, serían respectivamente los siguientes: 45,90 minutos/día, 68,27 minutos/día y 116,60 minutos/ día. Suponiendo que solo el grado o del BVD tiene traslación directa al Barthel (100 puntos), la dificultad se centra en precisar aquella puntuación Barthel que empíricamente iguale los tiempos medios de los grados I y II/III para Moretag (en función del índice Barthel) y para la normativa de referencia (ratios por grados del BVD). Según los resultados de la Tabla 3 para sucesivas reducciones en las puntuaciones Barthel a intervalos de cinco unidades, se alcanza un mínimo en la desviación del tiempo unitario (desviación absoluta igual o muy próxima a cero) entre los 55 y 60 puntos. Un intervalo que, por otra parte, es igualmente delimitado por ambas muestras $(\mathrm{N}=300$ y $\mathrm{N}=5.000)$ para un total de 30 simulaciones, resultado que ratifica la capacidad predictora de muestras relativamente pequeñas respecto del tamaño de la población de estudio.

\section{Interpretemos los datos obtenidos:}

- Cuando la puntuación frontera es de 58 puntos, la diferencia entre Moretag y la normativa de referencia es prácticamente del o \% en ambas muestras para el conjunto de simulaciones realizadas. En consecuencia, queda demostrado que el límite de 79 puntos, si bien eficaz para comprobar la dependencia entre Barthel y BVD desde el test de chi cuadrado, no es suficientemente verosímil para constituirse en disyuntiva de pertenencia al grado I o al grado II/ III, al menos en cuanto a tiempo medio de auxiliar de geriatría se refiere.

- La sensibilidad de la desviación entre tiempos de Moretag y tiempos de normativa apenas es apreciable hasta 75 puntos. A partir de esta puntuación, a cada intervalo constante de reducción le corresponde un decremento progresivo de la desviación hasta registrar un punto de inflexión para 58 puntos e IPD igual a 2,272 . Alcanzado su valor mínimo, la desviación crece exponencialmente a cada incremento marginal de la puntuación. Desde el cálculo del mínimo de la ecuación de regresión polinómica que correlaciona tiempo e IPD llegaríamos a una misma determinación analítica de la puntuación frontera ( 58,03 puntos). 
Tabla 3. Desviaciones entre tiempos medios de Moretag y tiempos medios según normativa de referencia(1)

\begin{tabular}{|c|c|c|c|c|c|c|c|c|c|}
\hline \multirow{3}{*}{$\begin{array}{l}\text { Puntos } \\
\text { frontera }\end{array}$} & \multicolumn{9}{|c|}{$\mathrm{N}=300$} \\
\hline & \multicolumn{3}{|c|}{$n=30$} & \multicolumn{3}{|c|}{$n=15$} & \multicolumn{3}{|c|}{$\mathrm{n}=7$} \\
\hline & IPD & Media & Desv. & IPD & Media & Desv. & IPD & Media & Desv. \\
\hline 95 & 2,540 & $-10,9 \%$ & $1,0 \%$ & 2,540 & $-10,9 \%$ & $0,9 \%$ & 2,540 & $-10,9 \%$ & $1,0 \%$ \\
\hline 90 & 2,540 & $-11,1 \%$ & $1,2 \%$ & 2,540 & $-11,0 \%$ & $1,2 \%$ & 2,540 & $-11,0 \%$ & $1,3 \%$ \\
\hline 85 & 2,540 & $-10,5 \%$ & $1,2 \%$ & 2,539 & $-10,5 \%$ & $1,3 \%$ & 2,539 & $-10,3 \%$ & $1,0 \%$ \\
\hline 80 & 2,536 & $-10,5 \%$ & $1,1 \%$ & 2,536 & $-10,7 \%$ & $1,3 \%$ & 2,535 & $-10,4 \%$ & $1,4 \%$ \\
\hline 75 & 2,518 & $-10,2 \%$ & $1,0 \%$ & 2,519 & $-10,2 \%$ & $1,1 \%$ & 2,520 & $-10,5 \%$ & $1,2 \%$ \\
\hline 70 & 2,488 & $-8,9 \%$ & $1,0 \%$ & 2,486 & $-8,9 \%$ & $1,0 \%$ & 2,492 & $-9,3 \%$ & $0,6 \%$ \\
\hline 65 & 2,473 & $-8,5 \%$ & $1,3 \%$ & 2,463 & $-8,2 \%$ & $1,5 \%$ & 2,427 & $-7,0 \%$ & $0,9 \%$ \\
\hline 60 & 2,297 & $-2,4 \%$ & $1,3 \%$ & 2,275 & $-2,2 \%$ & $1,1 \%$ & 2,233 & $-2,6 \%$ & $1,1 \%$ \\
\hline 55 & 2,173 & $4,0 \%$ & $1,4 \%$ & 2,175 & $3,7 \%$ & $1,4 \%$ & 2,182 & $3,1 \%$ & $1,3 \%$ \\
\hline 50 & 2,018 & $11,9 \%$ & $1,4 \%$ & 2,022 & $11,7 \%$ & $1,3 \%$ & 2,014 & $12,0 \%$ & $1,1 \%$ \\
\hline 45 & 1,848 & $22,4 \%$ & $1,6 \%$ & 1,854 & $22,2 \%$ & $1,8 \%$ & 1,844 & $22,1 \%$ & $1,7 \%$ \\
\hline
\end{tabular}

\begin{tabular}{|c|c|c|c|c|c|c|c|c|}
\hline \multicolumn{7}{|c|}{ N=5.000 } & \multicolumn{3}{c|}{ n=7 } \\
\hline IPD & Media & Desv. & IPD & Media & Desv. & IPD & Media & Desv. \\
\hline 2,540 & $-11,2 \%$ & $0,2 \%$ & 2,540 & $-11,2 \%$ & $0,2 \%$ & 2,540 & $-11,2 \%$ & $0,2 \%$ \\
\hline & & & 2,540 & $-11,3 \%$ & $0,2 \%$ & 2,540 & $-11,3 \%$ & $0,2 \%$ \\
\hline & & & & & & 2,539 & $-11,1 \%$ & $0,2 \%$ \\
\hline & & & & & & 2,535 & $-11,1 \%$ & $0,3 \%$ \\
\hline & & & & & & 2,521 & $-10,5 \%$ & $0,3 \%$ \\
\hline & & & & & & 2,486 & $-9,3 \%$ & $0,3 \%$ \\
\hline & & & & & & 2,422 & $-6,8 \%$ & $0,3 \%$ \\
\hline & & & & & & 2,321 & $-2,8 \%$ & $0,3 \%$ \\
\hline & & & & & & 2,182 & $3,2 \%$ & $0,4 \%$ \\
\hline & & & & & & 2,022 & $11,6 \%$ & $0,4 \%$ \\
\hline & & & & & & 1,850 & $21,8 \%$ & $0,6 \%$ \\
\hline
\end{tabular}

\begin{tabular}{|l|l|l|l|l|l|l|l|l|l|}
\hline 2,267 & $-0,5 \%$ & $0,3 \%$ & 2,266 & $-0,5 \%$ & $0,3 \%$ & 2,266 & $-0,6 \%$ & $0,3 \%$ \\
\hline
\end{tabular}

\begin{tabular}{|l|l|l|l|l|l|l|l|l|}
\hline 57,240 & $0,8 \%$ & $1,1 \%$ & 2,238 & $1,0 \%$ & $1,0 \%$ & 2,218 & $0,9 \%$ & $0,9 \%$ \\
\hline
\end{tabular}

\begin{tabular}{|l|l|l|l|l|l|l|l|l|}
\hline 2,239 & $0,8 \%$ & $0,2 \%$ & 2,239 & $0,8 \%$ & $0,2 \%$ & 2,239 & $0,8 \%$ & $0,2 \%$ \\
\hline
\end{tabular}

Decreto $38 / 2007$ de la Diputación Foral de Gipuzkoa.

No se realizan más de siete simulaciones porque se demuestra que no hay cambios sustanciales en los estadísticos a partir de dicho número.

Fuente: Elaboración propia.

- Aun cuando se demuestra que la frontera calculada minimiza la desviación, la amplitud del intervalo para grados II/III es tal (de o a 58 puntos Barthel) que el agrupamiento de perfiles en zonas bajas o altas del mismo supondría contar con horas por debajo o por encima de lo que propone Moretag en su versión original. Recordemos que Moretag determina tiempos conforme a la expresión que, a su vez, se descompone en tantos sumandos (perfiles del índice Barthel-CIF) como personas atendidas presente un centro residencial. Por su parte, las ratios de referencia para el ajuste de la pasarela Barthel-CIF producen un mínimo en términos medios que podría o no tener correspondencia con la suma de perfiles personalizados. Si los referidos perfiles se distribuyen uniformemente en el intervalo $[0,58]$, el efecto agregado desde Moretag original y desde Moretag según asociación Barthel-BVD sería muy similar. Por el contrario, si hubiera un sesgo claro hacia una de las dos cotas, la asignación de tiempos desde uno u otro método podría ofrecer resultados muy dispares (empíricamente se demuestra que para índices Barthel medios de 35 puntos o menos, Moretag por asociación tiende a estimar menores tiempos que Moretag original y que, además, lo hace en cuantía creciente a medida que disminuye dicha puntuación media). Dado que la dispersión es ciertamente más probable que el reparto uniforme de perfiles, se aconsejaría desdoblar el grado conjunto II/III en grado II y grado III para reducir los casos en los que la asociación Barthel-BVD produjera un número de horas inferior al propuesto por Moretag original. En su defecto, el debate quedaría abierto: introducir un factor corrector para la corrección de gaps o definir ratios estáticas para que la secuencia altabaja de residentes se produzca entre residentes de un mismo perfil. ¿Cómo afectarían ambas medidas a la gestión de las listas de espera?

Figura 4. Limitando la asociación Barthel-BVD

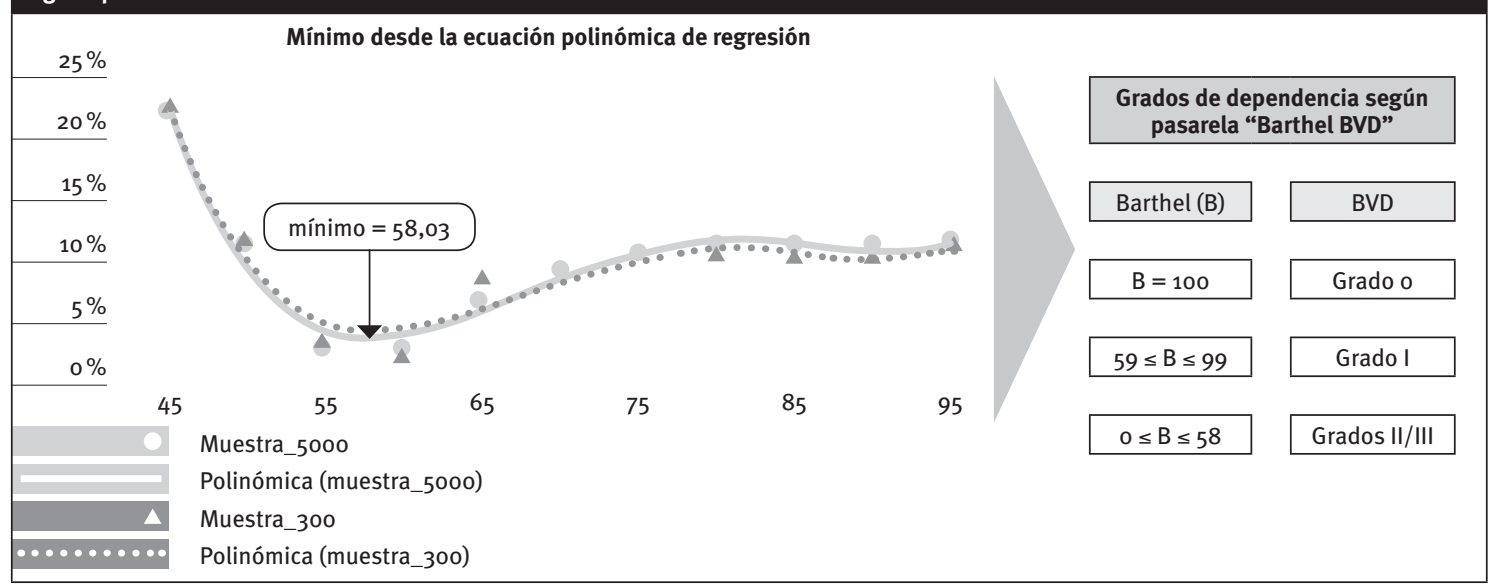

Fuente: Elaboración propia. 
3.3. Contraste entre los tiempos medios de cada normativa y los de Moretag adaptado desde el factor $p$

Recordemos que la expresión para el cálculo del factor de equivalencia es

$$
\mathrm{p}=\frac{\sum_{i=1}^{i=3} \mathrm{n}_{\mathrm{i}}{ }^{*} \mathrm{p}_{\mathrm{i}}}{\mathrm{n}},
$$

donde $n_{i}$ es el tamaño de cada estrato de dependencia y $p_{i}$ la adaptación teórica de las ratios de Moretag a las condiciones de cada normativa de contraste (ratios por grados de dependencia y jornada anuales en convenio colectivo). Recordemos, asimismo, que este factor referencia el cálculo sobre una hipotética proporcionalidad entre las diferentes tipologías de ratios - atención directa, $r_{\mathrm{ad}}$, $\mathrm{y}$ atención total, $r_{\mathrm{at}}-$ para cada estrato de dependencia cuando las normativas no especifican ratios de auxiliar de geriatría

$\left(\frac{\gamma_{g 2}}{\gamma_{g 1}} \cong \frac{\gamma_{a d}}{\gamma_{a d 1}} \cong \frac{\gamma_{a t 2}}{\gamma_{a t 1}}\right.$ y $\left.\frac{\gamma_{g 3}}{\gamma_{g 1}} \cong \frac{\gamma_{a d_{3}}}{\gamma_{a d_{1}}} \cong \frac{\gamma_{a t 3}}{\gamma_{a t 1}}\right)$.

El coeficiente $p_{i}\left(\mathrm{p}_{\mathrm{i}}=\frac{\mathrm{r}_{\mathrm{i}}}{\mathrm{r}_{\mathrm{r}-\mathrm{i}} \times \frac{J_{\mathrm{r}}}{\mathrm{J}}}\right.$, donde $\mathrm{r}_{\mathrm{i}} \mathrm{y}$ ) son,

respectivamente, la ratio del estrato $i$ y la jornada anual de trabajo en la normativa de contraste, $\mathrm{y}_{\mathrm{r}-\mathrm{i}}$ y $J_{r}$, idénticos conceptos en el modelo de referencia) estima el potencial de atención de cada normativa para cada estrato respecto de Moretag, patrón asistencial que despliega un catálogo de tareas directas e indirectas que se entiende troncal para el conjunto de centros residenciales del Estado. Si $p$ fuera inferior a uno, o se entiende una mayor productividad en las organizaciones de la normativa de contraste para conseguir idéntico nivel de atención que el propuesto por Moretag o, antes bien, para una misma capacidad productiva — nada argumenta una presunción en sentido contrario- el tiempo disponible para dar respuesta a un mismo catálogo de servicios es proporcionalmente inferior en la normativa de contraste o, asimismo, las tareas directas e indirectas previstas en Moretag no pueden ser ejecutadas a un mismo nivel de frecuencia $y / 0$ tiempo de atención. En cambio, si $p_{i}$ fuera superior a uno, la normativa de contraste podría incrementar la frecuencia, el tiempo y/o el abanico de tareas.

Veamos qué efectos produce el factor de equivalencia en los diferentes escenarios de atención, definidos como elementos del conjunto de posibles combinaciones de grados o, I y II/III ( $m i x$ de dependencia) para dos tamaños residenciales: 50 y 300 plazas. En ambos casos, el total de diferentes subgrupos que se pueden conformar como combinación de dichos grados es muy elevado (el despliegue total de trinomios o-I-II/III es una serie aritmética finita de diferencia igual a 3 que totaliza 3.978 combinaciones para 50 plazas y 136.353 para 300 plazas). Dada la presumible correlación entre el mix de dependencia (variable IPD) y el tiempo de auxiliar de geriatría, trabajaremos con combinaciones de grados de dependencia a intervalos de diez unidades, criterio que reduce sensiblemente el número de escenarios (21 para 50 plazas y 496 para 300 plazas) sin aparente menoscabo en la significación de los resultados obtenidos.

Partiendo de las ratios del modelo de referencia (0,175 para Barthel $=100,0,261$ para $59 \leq$ Barthel $\leq$ 99 y 0,446 para $0 \leq$ Barthel $\leq 58$ ), calculado el factor $p$ para cada normativa y aplicado para el conjunto de combinaciones consideradas, obtenemos la comparativa de la Tabla 4 para tiempos totales desde Moretag adaptado y desde las ratios actuales de cada normativa del Estado.

Figura 5. Desviaciones Moretag adaptado-normativa para los distintos niveles de IPD $(\mathrm{N}=300)$

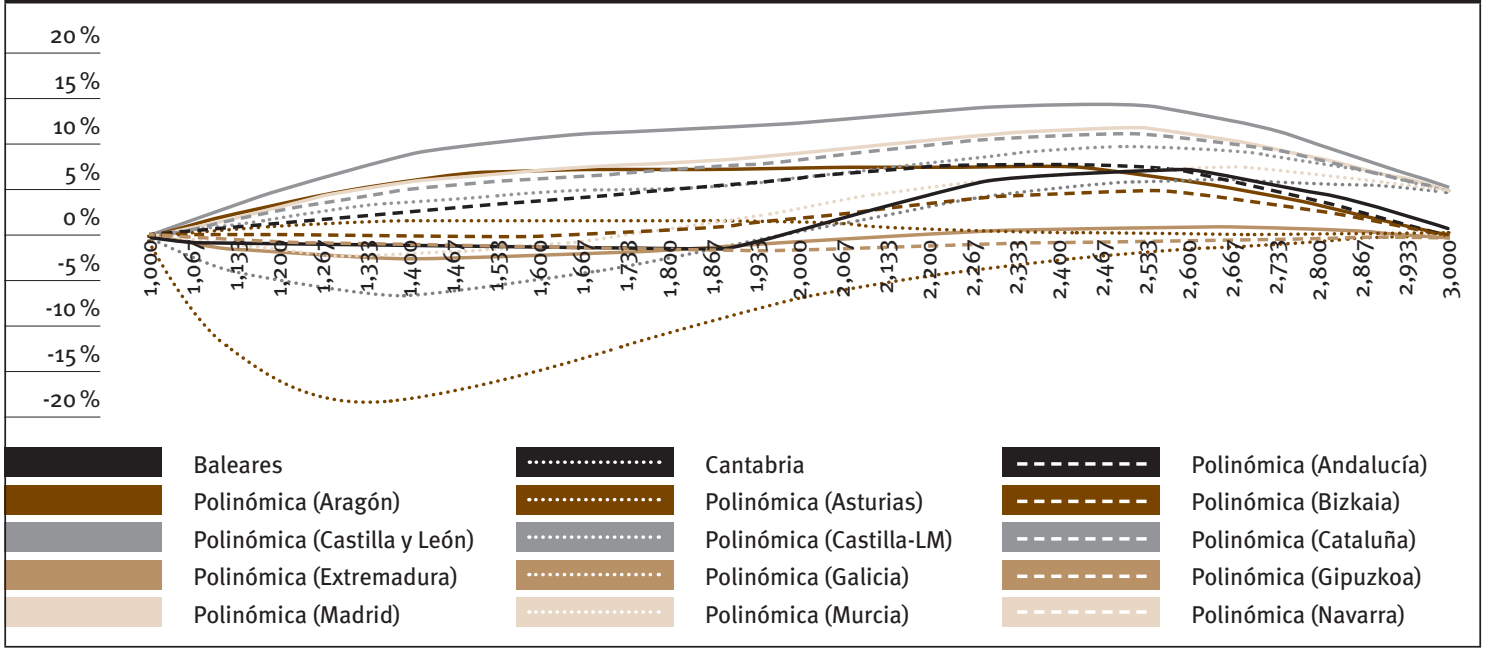

Interpretación del gráfico:

- El eje de abscisas contiene valores de IPD entre 1.000 y 3.000 como consecuencia de la adaptación de la expresión de IPD para representar homogéneamente, en función de una referencia compartida, el conjunto de normativas del Estado $\left(I P D=\frac{n_{1}+2 n_{2}+3 n_{3}}{n}\right)$.

- La correspondencia entre IPD y desviación norma-adaptación de Moretag desde factor p es ciertamente relevante para aquellas autonomías que discriminan ratios para los tres grados de dependencia (Asturias, Extremadura y Gipuzkoa).

Fuente: Elaboración propia. 
Según describe el gráfico de desviaciones, Moretag adaptado normalmente estima tiempos por encima de normativa en torno a 3 puntos de media $(2,9 \%$ para $\mathrm{N}=50$ y $3,3 \%$ para $\mathrm{N}=300$ ), aunque se registren desviaciones absolutas que incluso superan el $10 \%$ en determinados puntos del recorrido de IPD $\left(1 \leq \mathrm{IPD} \leq \mathrm{IPD}_{\max }\right)$. Si la curva de tiempos anuales de Moretag adaptado desde $p$ origina valores por encima/debajo relativamente extremos para calificarlos como tolerables, la curva de horas anuales según normativa debería experimentar un empuje en sentido contrario para comprimir la diferencia. Si no hubiera contigüidad mínimamente admisible (por ejemplo, en Castilla-León y Valencia y, en menor medida pero de desviación considerable, en Galicia, Cataluña y Madrid), las ratios deberían verse afectadas al alza para reducir la diferencia. El incremento, en cualquier caso, ha de conseguir que el tiempo total sea una función creciente en todo el recorrido de IPD y, en consecuencia, no origine máximo alguno para IPD $<I P D_{\max }$.
El factor $p$ debe producir resultados coherentes con la hipótesis de partida (proyección homogénea de Moretag para todos y cada uno de los valores de IPD). Cuando un centro cuenta en su totalidad con perfiles pertenecientes a un único grado de dependencia pero la normativa de aplicación especifica ratios para todos los grados, ambas referencias -Moretag y normativa - se igualan (desviación igual a cero) para volver a separarse en curvas que, en determinadas normativas, alcanzan tal nivel de divergencia que podrían cuestionar la validez del método. Por tanto, si buscamos homogeneizar el contraste entre normativas para dotar de coherencia al modelo, el objetivo buscado no es otro que conseguir un nivel de paralelismo suficiente entre tiempos según normativa y tiempos de Moretag adaptado; en otras palabras, las ratios de las normativas deberían ser tales que redujeran las diferencias para cada IPD entre las horas anuales derivadas de aquellas y las propuestas por Moretag adaptado. ¿Desde qué ecuación conseguiremos aproximar ambas curvas hasta extremos de tolerancia suficiente?

Tabla 4. Diferencias entre Moretag adaptado y normativas del Estado

\begin{tabular}{|l|c|c|c|}
\hline \multirow{2}{*}{ Normativa } & \multicolumn{3}{|c|}{ Ratios $^{(1)}$} \\
\cline { 2 - 4 } & \multicolumn{1}{|c|}{ I } & I & IIII \\
\hline Andalucía & & & \\
\hline Asturias & 0,125 & 0,200 & 0,200 \\
\hline Castilla-LM & 0,120 & 0,150 & 0,275 \\
\hline Extremadura & 0,067 & 0,167 & 0,167 \\
\hline Gipuzkoa & 0,050 & 0,100 & 0,143 \\
\hline Valencia & 0,149 & 0,239 & 0,420 \\
\hline Baleares & 0,033 & 0,242 & 0,306 \\
\hline La Rioja & & 0,300 & 0,300 \\
\hline Araba & & & 0,280 \\
\hline Bizkaia & 0,163 & 0,293 & 0,293 \\
\hline Castilla y León & 0,163 & 0,293 & 0,293 \\
\hline Galicia & 0,219 & 0,237 & 0,237 \\
\hline Murcia & 0,200 & 0,350 & 0,350 \\
\hline Cantabria & 0,100 & 0,250 & 0,250 \\
\hline Aragón & & 0,295 & 0,337 \\
\hline Cataluña & 0,250 & 0,300 & 0,350 \\
\hline Madrid & 0,250 & 0,370 & 0,370 \\
\hline Navarra & 0,250 & 0,350 & 0,350 \\
\hline Canarias & 0,100 & 0,350 & 0,350 \\
\hline Estadísticos medios del Estado & & 0,460 \\
\hline & & & \\
\hline
\end{tabular}

\begin{tabular}{|c|c|}
\hline \multicolumn{2}{|c|}{$\mathbf{N =} \mathbf{5 0}^{(2)}$} \\
(21 combinaciones) \\
\hline Media & Desviación \\
\hline $3.8 \%$ & $3,0 \%$ \\
\hline $0,7 \%$ & $0,8 \%$ \\
\hline $0,8 \%$ & $3,2 \%$ \\
\hline$-0,6 \%$ & $1,4 \%$ \\
\hline$-0,7 \%$ & $0,5 \%$ \\
\hline$-6,0 \%$ & $6,9 \%$ \\
\hline $3,9 \%$ & $3,2 \%$ \\
\hline & \\
\hline $4,7 \%$ & $3,7 \%$ \\
\hline $4,7 \%$ & $3,7 \%$ \\
\hline $8,6 \%$ & $4,6 \%$ \\
\hline $4,9 \%$ & $3,6 \%$ \\
\hline $2,5 \%$ & $4,4 \%$ \\
\hline $2,6 \%$ & $2,1 \%$ \\
\hline $4,5 \%$ & $2,9 \%$ \\
\hline $6,1 \%$ & $3,7 \%$ \\
\hline $6,5 \%$ & $3,8 \%$ \\
\hline $0,6 \%$ & $5,5 \%$ \\
\hline $2,9 \%$ & $2,5 \%$ \\
\hline
\end{tabular}

\begin{tabular}{|c|c|}
\hline \multicolumn{2}{|c|}{$\begin{array}{c}\mathrm{N}=300^{(2)} \\
\text { (496 combinaciones) }\end{array}$} \\
\hline Media & Desviación \\
\hline $5,8 \%$ & $3,1 \%$ \\
\hline $1,0 \%$ & $0,9 \%$ \\
\hline $1,4 \%$ & $2,8 \%$ \\
\hline$-0,7 \%$ & $1,4 \%$ \\
\hline$-1,1 \%$ & $0,6 \%$ \\
\hline$-8,3 \%$ & $7,3 \%$ \\
\hline $4,7 \%$ & $2,4 \%$ \\
\hline $6,1 \%$ & $3,5 \%$ \\
\hline $6,1 \%$ & $3,5 \%$ \\
\hline $11,7 \%$ & $5,7 \%$ \\
\hline $6,3 \%$ & $3,6 \%$ \\
\hline $2,9 \%$ & $3,5 \%$ \\
\hline $3,1 \%$ & $1,6 \%$ \\
\hline $6,6 \%$ & $3,3 \%$ \\
\hline $8,1 \%$ & $4,1 \%$ \\
\hline $8,7 \%$ & $4,3 \%$ \\
\hline $0,2 \%$ & $4,5 \%$ \\
\hline $3,3 \%$ & $3,3 \%$ \\
\hline
\end{tabular}

(1) Para poder estimar horas anuales de auxiliar de geriatría “comparables" para normativas que no exigen ratios de gerocultor/a, el modelo infiere el valor de las ratios de atención directa o, en su defecto, de atención total conforme a la proporcionalidad existente entre las referidas ratios para cada grado de dependencia en el modelo de referencia.

(2) Una vez más, las diferencias medias a partir de un determinado número de observaciones no son estadísticamente significativas (desviación media total igual a $2,9 \%$ para $\mathrm{N}=50$ y a $3,3 \%$ para $\mathrm{N}=300$ ).

(3) Habida cuenta del plazo de dos años previsto por la disposición transitoria primera del recientemente aprobado Decreto $126 / 2019$ para la adecuación de los centros residenciales en funcionamiento, los cálculos recogidos en esta tabla y posteriores se referencian en el decreto 41/1998 del Gobierno Vasco y no en las nuevas ratios, a día de hoy no exigibles con efecto inmediato.

Fuente: Elaboración propia. 


\subsubsection{Minimizando la desviación conjunta entre normativa y Moretag adaptado}

Las horas totales son la suma del producto de tres factores: número de residentes por grado de dependencia, ratio de auxiliar de geriatría (directa o estimada según proporcionalidad entre otras ratios) y jornada anual establecida por convenio colectivo. Moretag adaptado y normativa presentarán curvas prácticamente solapadas cuando la legislación correspondiente concreta ratios de gerocultor/a respetando la relación $r_{1} \leq r_{2} \leq r_{3}$, por otra parte únicamente operativa para tres normativas del Estado: Asturias, Extremadura y Gipuzkoa. Para estas se cumple que el tiempo total es función directa del IPD: incrementos marginales en el valor de IPD producen aumentos de diferente pendiente en la curva de tiempos totales. Para el resto de normativas, la adaptabilidad de Moretag desde el factor $p$, aun siendo consistente en la mayoría de casos analizados, presenta discordancias presumiblemente corregibles. Referir dicho factor desde ratios de atención directa o total, máxime si no se distinguen grados de dependencia, tiende a acrecentar el diferencial entre Moretag y normativa. En otros términos, no todas las normativas concretan ratios tan armónicas como para validarlas desde Moretag conforme a intervalos de desviación absoluta tolerable. El panorama estatal, excluidas las normas cuya desviación promedio es de apenas un $1 \%(1,13 \%$ para Asturias, $1,36 \%$ para Extremadura y 1,06\% para Gipuzkoa), presenta los estadísticos de la Tabla 4. Para que el gap Moretag-normativa vea reducido su valor medio, las actuales ratios en normativa deberían adoptar otros valores (incremento para normativas con desviaciones positivas y decremento para las negativas).

Hasta el momento solo hemos estimado la adaptación de Moretag para un tamaño de muestra compuesto por 496 observaciones (combinaciones de grados o, I y II/III para un centro de 300 plazas). Siendo absolutamente puristas, Moretag y normativa deberían coincidir cualquiera que fuera el valor de IPD, una variable "discreta" que, para normativas con ratios para cada grado de dependencia, presentaría tantos valores como combinaciones hubiéramos identificado. Matemáticamente expresado, cada punto de igualdad sería, a su vez, una ecuación que igualara Moretag y normativa con nuevas ratios. En conjunto, deberíamos resolver un sistema de $N$ ecuaciones $(N=496)$ con tres incógnitas $\left(r_{1}, r_{2}\right.$ y $r_{3}$ ) para normativas que discriminan ratios por grado y de 31 ecuaciones con dos incógnitas $\left(r_{2}\right.$ $\mathrm{y} \mathrm{r}_{3)}$ para aquellas que no contemplan grado $o$. En ambos casos, presumimos que el sistema es irresoluble al estar compuesto por tres ecuaciones que directamente determinarían el valor de $r_{1}, r_{2}$ y $r_{3}$ que, además, por la composición de la expresión que calcula el factor $p$ de equivalencia, no producirían ratios distintas a las ya existentes.

Deducimos, por tanto, que la expresión para el cálculo de ratios que minoren las desviaciones observadas debe ser planteada desde el cumplimiento de condiciones alternativas. Dado, por un lado, el considerable tamaño de la muestra y, por otro, la sensibilidad marginal de la variable IPD como consecuencia del reparto proporcional de grados en aquella, podrían plantearse otros procedimientos de cálculo igualando la suma de los tiempos totales para cada IPD desde la adaptación de Moretag según el factor $p$ y desde la normativa de contraste afectada por una tasa de variación en sus ratios actuales. Para la fijación de la tasa, constante para cada grado de dependencia o variable en función de la proporcionalidad entre ratios, resolveríamos las siguientes ecuaciones:

- Fijación constante: la suma total de los tiempos totales para cada IPD según Moretag adaptado desde $p$ será igual a la suma total de los tiempos totales según ratios de normativa variadas en función de la tasa calculada.

- Fijación variable: la suma total de los tiempos totales para cada IPD según Moretag adaptado desde $p$ será igual a la suma total de los tiempos totales según ratios de normativa variadas en función de la tasa de cada grado como consecuencia de la proporcionalidad existente entre las ratios de dichos grados.

Aunque una y otra alternativa produzcan resultados muy similares, la fijación proporcional presenta una desviación media total algo inferior y, por tanto,

Tabla 5. Ejemplo de ecuaciones para cálculo directo de $r_{1}, r_{2}$ y $r_{3}$ (Asturias)

\begin{tabular}{|c|c|c|c|c|c|c|c|}
\hline \multicolumn{3}{|c|}{ Ratios } & \multicolumn{3}{|c|}{ Mix de dependencia } & \multirow[b]{2}{*}{ Moretag adaptado } & \multirow[b]{2}{*}{ Ecuación y cálculo de nuevas ratios } \\
\hline $\begin{array}{c}\text { Grado } \\
0\end{array}$ & $\begin{array}{c}\text { Grado } \\
\text { I }\end{array}$ & $\begin{array}{l}\text { Grado } \\
\text { II/III(*) }\end{array}$ & $\begin{array}{c}\text { Grado } \\
0\end{array}$ & $\begin{array}{c}\text { Grado } \\
\text { I }\end{array}$ & $\begin{array}{l}\text { Grado } \\
\text { II/III }\end{array}$ & & \\
\hline 0,120 & 0,150 & 0,275 & 300 & 0 & 0 & $\frac{0,120}{0,175 \frac{1.592}{1.792}} * 83.580=64.512$ & $64 \cdot 512=r_{1}{ }^{*} 1.792 * 300 \longrightarrow r_{1}=0,120$ \\
\hline & & & 0 & 300 & 0 & $\frac{0,150}{0,261 \frac{1.592}{1.792}} * 107.520=80.640$ & $80.640=r_{2}{ }^{*} 1.792 * 300 \longrightarrow r_{2}=0,150$ \\
\hline & & & 0 & 0 & 300 & $\frac{0,275}{0,446 \frac{1.592}{1.792}} * 213.010=147.840$ & $147-840=r_{3}{ }^{*} 1.792 * 300 \longrightarrow r_{3}=0,275$ \\
\hline
\end{tabular}

$\left.{ }^{\star}\right)$ Media de ratios de grado II $(0,270)$ y de grado III $(0,280)$

Fuente: Elaboración propia. 
prácticamente yuxtapone las curvas de tiempos totales desde Moretag adaptado y desde la normativa afectada por las ratios de aproximación. En ambos casos, prácticamente la totalidad de las normativas del Estado ven reducidas sus desviaciones medias hasta valores próximos a cero, resultado que parece validar el método de cálculo en la medida en que minimizamos las desviaciones medias. No obstante, ambas expresiones adolecerían de un problema recurrente si se aplicaran en modo sucesivo. Si por efecto del cálculo se definen nuevas ratios que igualen la suma de tiempos totales para cada IPD desde Moretag - adaptado conforme a las ratios previas al cálculo-y desde normativa - de acuerdo con las nuevas ratios-, el modelo propondría nuevas adaptaciones porque, precisamente, las ratios se han visto afectadas. Teóricamente, esta recurrencia implícita dejaría de reproducirse cuando cada normativa contuviera idénticas ratios que Moretag en su versión original (0,175, 0,261 y 0,446 respectivamente para cada grado de dependencia), consecuencia más que improbable habida cuenta del actual panorama estatal. En consecuencia, su validez quedaría limitada al primer estadio de cálculo o aquel referenciado sobre las ratios vigentes, cuyo valor de referencia no debería experimentar cambio alguno salvo nueva definición de ratios en Moretag y/o en la normativa correspondiente.

Dada la reciprocidad subyacente en el método empleado interesaría imaginar alguna otra alternativa de cálculo que supusiera, en sí misma, un principio y un fin. Para cumplir con esta condición, el factor de equivalencia debería contemplar el valor de las ratios como variables y no como parámetros. De modo similar a la fijación constante o proporcional, la suma total de tiempos de Moretag adaptado debería ser igual a la suma de tiempos de normativa para cada combinación de dependencia en la muestra de 496 elementos. En este caso, en lugar de calcular tasas de variación de las ratios existente calcularemos directamente el valor de las mismas. El razonamiento matemático deriva en un sistema de tres ecuaciones con tres incógnitas $\left(r_{1}, r_{2}\right.$ y $\left.r_{3}\right)$ que fracciona el recorrido de IPD en tres tramos, correspondiente cada uno de ellos a un tercio de las 496 combinaciones. Ilustremos el método desde las ratios correspondientes a la normativa de Andalucía (Orden 1 de julio de 1997, por la que se regula la acreditación de centros de atención especializada para las personas mayores y las personas con discapacidad, Boletín Oficial de la Junta de Andalucía, 81):

$$
\left\{\begin{array}{l}
16.486 .475 r_{1}-2.303 .514 r_{2}-5.120 .888 r_{3}=0 \\
15.957 .862 r_{1}+2.713 .788 r_{2}-7.849 .609 r_{3}=0 \\
2.4790 .658 r_{1}+15.781 .730 r_{2}-4.204 .246 r_{3}=0
\end{array}\right.
$$

Obsérvese que el sistema obtenido es homogéneo y compatible (siempre existe la solución $r_{1}=0, r_{2}$ $=0 \mathrm{y}_{3}=0$ ) que, resuelto conforme al método de reducción de Gauss, ofrece infinitas soluciones

Tabla 6. Comparativa de desviaciones medias según alternativa

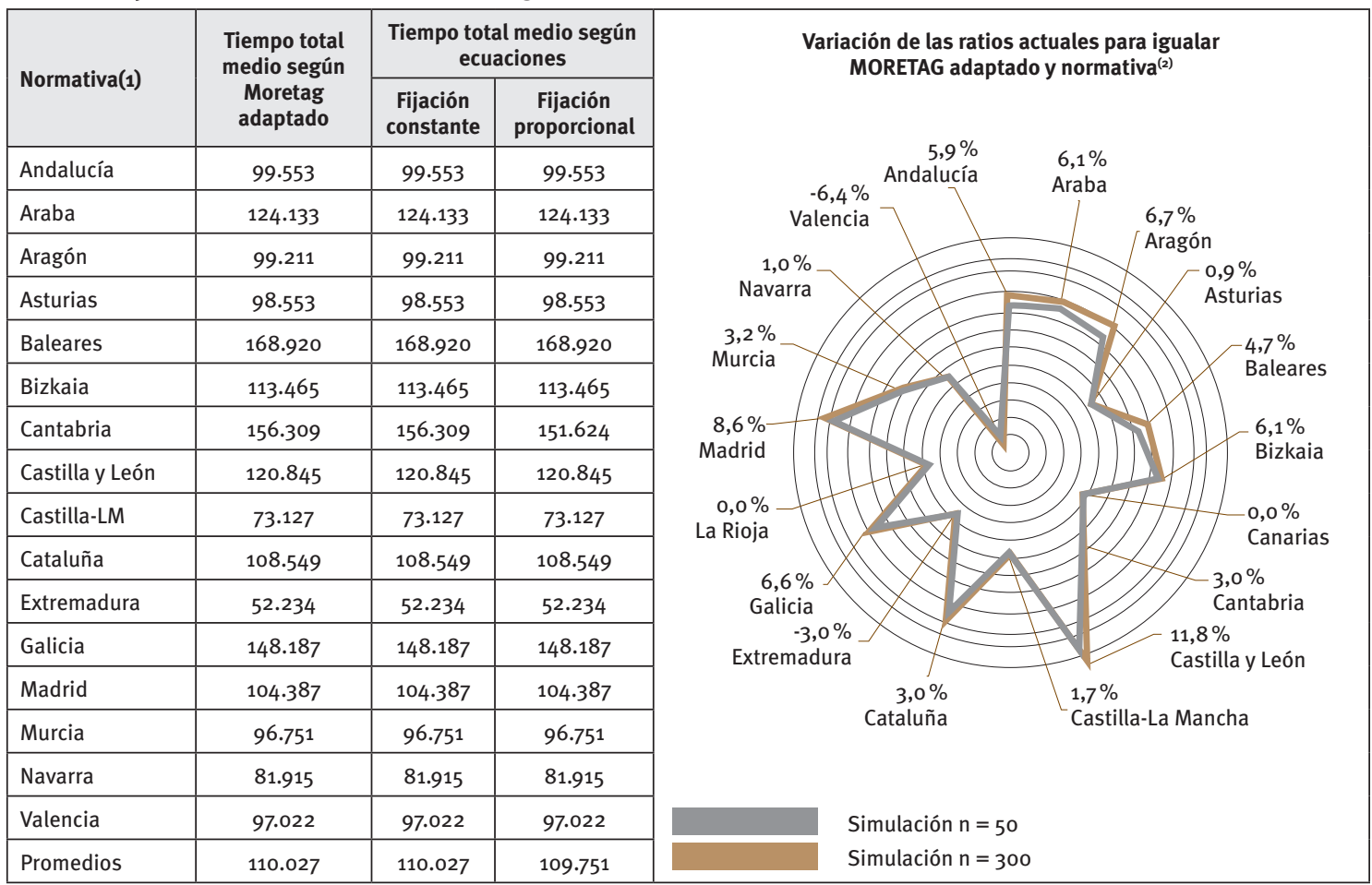

(1) - Obsérvese que Gipuzkoa no presenta Moretag adaptado. La razón no es otra que obviar la comparativa de una normativa sobre sí misma habida cuenta de que Moretag y Gipuzkoa confluyen prácticamente en una misma referencia.

- Canarias y La Rioja presentan valores nulos porque solo discriminan ratios para grados II-III y, por esta razón, no se presentan datos.

(2) Nuevamente se demuestra la capacidad predictora de muestras pequeñas.

Fuente: Elaboración propia. 
desde la parametrización de una de sus variables. Tampoco esta alternativa parece adecuada por cuanto de apriorismo supone la previa asignación de valor a una de las ratios para calcular las restantes. En consecuencia, deberíamos transformar el sistema para obtener otro que, partiendo de la ratio correspondiente al grado o, obtenga el valor de las ratios de grado I y grados II/III que iguale las sumas de tiempos totales desde Moretag adaptado y desde normativa.

Dada la supuesta relación $r_{1} \leq r_{2} \leq r_{3}$, que, según demuestra Moretag, debiera caracterizar la asignación de ratios, procede tomar el grado o como referencia para la cuantificación de los dos términos independientes del sistema. Empíricamente se evidencia que la presencia de este estrato es residual en los centros residenciales y, en consecuencia, interesa calcular la ratio del grado predominante (II/III) y su variación respecto del valor actual para poder cotejar la supuesta relación entre el tiempo de auxiliar y el nivel de dependencia. Una vez parametrizada la ratio de grado o, se obtiene un sistema lineal de dos ecuaciones con dos incógnitas correspondientes, respectivamente, a cada mitad del recorrido de IPD (observaciones 1 a 248 y 249 a 496). Sigamos con el ejemplo andaluz para reducir el sistema:

$$
\left\{\begin{array}{l}
1.806 .148 r_{2}+1.034 .7421 r_{3}=3.680 .087 \\
-10.015 \cdot 518 r_{2}+13.180 .307 r_{3}=2.331 .691
\end{array}\right.
$$

El cálculo de $r_{2}$ y $r_{3}$, si bien respeta la relación $r_{1}$ $\leq r_{2} \leq r_{3}$ para las normativas que especifican ratios para grados o y I - por asimilación, grados II/III-y, asimismo, iguala las sumas totales de tiempos desde Moretag y desde el valor de aquellas, plantearía tal cambio de escenario que muchos ejecutivos autonómicos abandonarían cualquier iniciativa de cambio por inasumible. Tan solo Araba, Bizkaia y Galicia presentan suficiente coherencia en los resultados obtenidos para considerar que Moretag adaptado desde sistemas de ecuaciones puede erigirse en un referente fiable para la asignación homogénea de ratios.

Tabla 7. Comparativa de desviaciones por resolución de sistema de ecuaciones

\begin{tabular}{|c|c|c|c|c|}
\hline \multirow{2}{*}{ Normativa } & \multirow{2}{*}{ Referencia } & \multicolumn{3}{|c|}{ Ratios actuales } \\
\hline & & o & I & II-III \\
\hline Andalucía & Gerocultor/a & 0,125 & 0,200 & 0,200 \\
\hline Araba & $\begin{array}{l}\text { Atención } \\
\text { directa }\end{array}$ & 0,143 & 0,255 & 0,255 \\
\hline Aragón & Atención total & 0,142 & 0,170 & 0,208 \\
\hline Asturias & Gerocultor/a & 0,120 & 0,150 & 0,275 \\
\hline Baleares & Gerocultor/a & & 0,300 & 0,300 \\
\hline Bizkaia & $\begin{array}{l}\text { Atención } \\
\text { directa }\end{array}$ & 0,143 & 0,255 & 0,255 \\
\hline Canarias & Atención total & & & 0,273 \\
\hline Cantabria & $\begin{array}{l}\text { Atención } \\
\text { directa }\end{array}$ & & 0,256 & 0,308 \\
\hline $\begin{array}{l}\text { Castilla y } \\
\text { León }\end{array}$ & $\begin{array}{l}\text { Atención } \\
\text { directa }\end{array}$ & 0,192 & 0,206 & 0,206 \\
\hline Castilla-LM & Gerocultor/a & 0,067 & 0,167 & 0,167 \\
\hline Cataluña & $\begin{array}{l}\text { Atención } \\
\text { directa }\end{array}$ & 0,142 & 0,209 & 0,209 \\
\hline Extremadura & Gerocultor/a & 0,050 & 0,100 & 0,143 \\
\hline Galicia & $\begin{array}{l}\text { Atención } \\
\text { directa }\end{array}$ & 0,175 & 0,304 & 0,304 \\
\hline Gipuzkoa & Gerocultor/a & 0,149 & 0,239 & 0,420 \\
\hline La Rioja & Gerocultor/a & & & 0,280 \\
\hline Madrid & Atención total & 0,142 & 0,198 & 0,198 \\
\hline Murcia & $\begin{array}{l}\text { Atención } \\
\text { directa }\end{array}$ & 0,088 & 0,217 & 0,217 \\
\hline Navarra & Atención total & 0,057 & 0,198 & 0,198 \\
\hline Valencia & Gerocultor/a & 0,033 & 0,242 & 0,306 \\
\hline
\end{tabular}

\begin{tabular}{|c|c|c|c|c|c|}
\hline \multicolumn{6}{|c|}{ Resultados para las 496 combinaciones de la muestra } \\
\hline \multicolumn{3}{|c|}{ Ratios propuestas } & \multicolumn{3}{|c|}{ Tiempos totales medios } \\
\hline o & $\mathbf{I}$ & II-III & Norma actual & Sistema ${ }^{(2)}$ & Variación \\
\hline 0,125 & 0,191 & 0,322 & 94.080 & 114.330 & $21,5 \%$ \\
\hline 0,125 & 0,190 & 0,336 & 116.866 & 116.779 & $-0,1 \%$ \\
\hline 0,081 & 0,123 & 0,218 & 93.060 & $75 \cdot 558$ & $-18,8 \%$ \\
\hline 0,120 & 0,184 & 0,309 & 97.664 & 109.850 & $12,5 \%$ \\
\hline 0,125 & 0,190 & 0,336 & 106.822 & 106.743 & $-0,1 \%$ \\
\hline 0,168 & 0,256 & 0,452 & 108.218 & 156.935 & $45,0 \%$ \\
\hline 0,067 & 0,103 & 0,173 & 71.859 & 61.466 & $-14,5 \%$ \\
\hline 0,081 & 0,123 & 0,218 & 100.403 & $75 \cdot 558$ & $-24,7 \%$ \\
\hline 0,050 & 0,077 & 0,129 & 52.506 & 45.875 & $-12,6 \%$ \\
\hline 0,153 & 0,233 & 0,413 & 139.178 & 141.901 & $2,0 \%$ \\
\hline 0,149 & 0,228 & 0,384 & 128.634 & 121.151 & $-5,8 \%$ \\
\hline 0,081 & 0,123 & 0,218 & 96.030 & 75.305 & $-21,6 \%$ \\
\hline 0,077 & 0,116 & 0,206 & 93.577 & 71.668 & $-23,4 \%$ \\
\hline 0,032 & 0,049 & 0,087 & 81.066 & 30.245 & $-62,7 \%$ \\
\hline 0,033 & 0,050 & 0,085 & 103.650 & 29.971 & $-71,1 \%$ \\
\hline & omedio & & 98.908 & 88.889 & $-11,63 \%$ \\
\hline
\end{tabular}

(1) Cuando la normativa no especifica ratios de gerocultor/a, se estiman conforme a la proporcionalidad existente entre los tres tipos de ratio (gerocultor/a, directa y total) en la normativa que fundamenta Moretag (Decreto Foral 38/2007 de Diputación Foral de Gipuzkoa).

(2) La desviación entre el sistema y, por ende, entre las ratios propuestas para cada normativa y la adaptación de Moretag en función de estas es prácticamente nula en todos los casos.

Fuente: Elaboración propia. 
Por todo ello, podemos concluir que:

- La determinación de ratios desde sistemas de ecuaciones es un método adecuado para igualar Moretag adaptado y normativa que, además, cumple con la hipótesis de tiempos totales crecientes en función del IPD. Sin embargo, no es verosímil porque se aleja considerablemente de la realidad.

- En sí mismo, el factor de equivalencia $p$ se perfila como un buen adaptador. No origina escenarios inalcanzables y permitiría un progresivo acercamiento entre normativa y Moretag desde la sucesiva consolidación de aquellas ratios que minimizan la desviación entre ambas para cada fase de aproximación. Con el método de fijación de tasas de variación sobre las ratios operativas podríamos planificar la velocidad con la que cada ejecutivo, considerado Moretag como una referencia suficientemente válida por rigurosa y transversal, pretende asimilarse al modelo diseñado por Lares Euskadi.

\subsubsection{Panorama estatal (dispersión), verosimilitud (minimización) y homogeneización (adaptación)}

La divergencia es probablemente la característica que mejor describe cualquier análisis sobre el conjunto de normativas del Estado. Sin embargo, si es consecuencia del posicionamiento de cada Gobierno autonómico o territorial en uso de sus propias competencias administrativas, poco cabe hacer al respecto salvo homogeneizar, en la medida de lo teóricamente posible, las comparativas entre unas y otras. En ningún caso y bajo ningún pretexto, el presente estudio busca ensalzar el modelo guipuzcoano como principio rector de nada. Tan solo se hace eco de la evidente paridad entre Moretag y el Decreto 38/2007 de la Diputación Foral de Gipuzkoa y, consecuentemente, lo dota de sentido referencial para el diseño de un marco teórico de asimilación de ratios de gerocultor/a. Porque, si bien tanto la calidad como la cantidad de atención están inequívocamente vinculadas a la disponibilidad de recursos -entre ellos, sustancialmente, las y los auxiliares de geriatría-, nada argumenta que a mayor ratio, mayores garantías para atender en clave de perspectiva humanista.

Los factores intangibles en la provisión de servicios sociales o aquellos que Martin Knapp (1988) considera implícitos en las personalidades, actividades, actitudes y experiencias de los principales actores del proceso asistencial y en especial del personal de atención directa y de las personas residentes, son tanto o más garantes de una adecuada atención que los factores tangibles (bienes de equipo, personal o materias primas). Por tanto, podría ocurrir que aquello de que “menos

Tabla 8. Comparativa normativa y Moretag adaptado (fijación proporcional) para una residencia de cien plazas

Distribución de plazas por grados de dependencia

\begin{tabular}{|l|c|c|c|c|c|c|}
\hline \multirow{2}{*}{ Normativa } & \multicolumn{2}{|c|}{ Ratios en norma } & \multicolumn{3}{|c|}{$\begin{array}{c}\text { Ratios de } \\
\text { adaptación }\end{array}$} \\
\cline { 2 - 7 } & 0 & I & II/III & 0 & I & II/III \\
\hline Gipuzkoa & 0,149 & 0,239 & 0,420 & & & \\
\hline Andalucía & 0,125 & 0,200 & 0,200 & 0,132 & 0,212 & 0,212 \\
\hline Araba & 0,163 & 0,293 & 0,293 & 0,173 & 0,311 & 0,311 \\
\hline Aragón & 0,250 & 0,300 & 0,350 & 0,267 & 0,320 & 0,373 \\
\hline Asturias & 0,120 & 0,150 & 0,275 & 0,121 & 0,151 & 0,278 \\
\hline Baleares & & 0,300 & 0,300 & & 0,314 & 0,314 \\
\hline Bizkaia & 0,163 & 0,293 & 0,293 & 0,173 & 0,311 & 0,311 \\
\hline Canarias(*) & & & 0,460 & & & \\
\hline Cantabria & & 0,295 & 0,337 & & 0,304 & 0,347 \\
\hline $\begin{array}{l}\text { Castilla y } \\
\text { León }\end{array}$ & 0,219 & 0,237 & 0,237 & 0,245 & 0,265 & 0,265 \\
\hline Castilla-LM & 0,067 & 0,167 & 0,167 & 0,068 & 0,170 & 0,170 \\
\hline Cataluña & 0,250 & 0,370 & 0,370 & 0,270 & 0,400 & 0,400 \\
\hline Extrem. & 0,050 & 0,100 & 0,143 & 0,050 & 0,099 & 0,142 \\
\hline Galicia & 0,200 & 0,350 & 0,350 & 0,213 & 0,373 & 0,373 \\
\hline La Rioja(*) & & & 0,280 & & & \\
\hline Madrid & 0,250 & 0,350 & 0,350 & 0,272 & 0,380 & 0,380 \\
\hline Murcia & 0,100 & 0,250 & 0,250 & 0,103 & 0,258 & 0,258 \\
\hline Navarra & 0,100 & 0,350 & 0,350 & 0,101 & 0,354 & 0,354 \\
\hline Valencia & 0,033 & 0,242 & 0,306 & 0,031 & 0,227 & 0,286 \\
\hline
\end{tabular}

\begin{tabular}{|c|c|c|}
\hline 0 & I & II/III \\
\hline 80 & 20 & 0 \\
\hline
\end{tabular}

\begin{tabular}{|l|l|l|l|}
\hline \multirow{2}{*}{ IPD } & \multirow{2}{*}{$\begin{array}{c}\text { Horas } \\
\text { según } \\
\text { norma }\end{array}$} & \multicolumn{2}{|c|}{$\begin{array}{c}\text { Moretag } \\
\text { adaptado }\end{array}$} \\
\cline { 3 - 4 } & & Horas & Desv. \\
\hline & 26.586 & & \\
\hline & & & \\
\hline
\end{tabular}

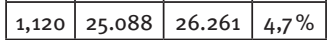

\begin{tabular}{|l|l|l|l|}
1,160 & 29.640 & 31.026 & $4,7 \%$ \\
\hline
\end{tabular}

\begin{tabular}{|l|l|l|l|}
\hline 1,040 & 26.450 & 27.922 & $5,6 \%$ \\
\hline
\end{tabular}

\begin{tabular}{|l|l|l|l|}
\hline 1,050 & 22.579 & 22.706 & $0,6 \%$ \\
\hline
\end{tabular}

\begin{tabular}{|l|l|l|l|}
\hline 10.752 & 11.261 & $4,7 \%$ \\
\hline
\end{tabular}

\begin{tabular}{l|l|l|l|}
\hline 1,160 & 27.093 & 28.360 & $4,7 \%$ \\
\hline
\end{tabular}

\begin{tabular}{|c|c|c|c|}
\hline 1,160 & 27.093 & 28.360 & $4,7 \%$ \\
\hline & 0 & 0 & $0,0 \%$ \\
\hline & 9.175 & 9.189 & $0,1 \%$ \\
\hline
\end{tabular}

\begin{tabular}{l|l|l|l|}
1,016 & 34.908 & 38.838 & $11,3 \%$
\end{tabular}

\begin{tabular}{l|l|l|l|}
\hline 1,299 & 15.590 & 15.783 & $1,2 \%$ \\
\hline
\end{tabular}

\begin{tabular}{|l|l|l|l|}
\hline 1,096 & 27.848 & 29.761 & $6,9 \%$ \\
\hline
\end{tabular}

\begin{tabular}{|l|l|l|l|}
\hline 1,200 & 10.752 & 10.719 & $-0,3 \%$ \\
\hline
\end{tabular}

\begin{tabular}{|l|l|l|l|}
1,150 & 35.662 & 37.509 & $5,2 \%$ \\
\hline
\end{tabular}

\begin{tabular}{|c|c|c|c|} 
& 0 & 0 & $0,0 \%$ \\
\hline 1,080 & 27.362 & 29.415 & $7,5 \%$ \\
\hline
\end{tabular}

\begin{tabular}{|l|l|l|l|}
\hline 1,300 & 20.393 & 20.837 & $2,2 \%$ \\
\hline
\end{tabular}

\begin{tabular}{l|l|l|l|}
1,500 & 15.268 & 15.349 & $0,5 \%$ \\
\hline
\end{tabular}

\begin{tabular}{|l|l|l|l|}
\hline 2,267 & 13.344 & 12.800 & $-4,1 \%$ \\
\hline
\end{tabular}

\begin{tabular}{|c|c|c|}
\hline 0 & I & II/III \\
\hline 33 & 33 & 34 \\
\hline
\end{tabular}

\begin{tabular}{|c|c|c|c|}
\hline \multirow{2}{*}{ IPD } & \multirow{2}{*}{$\begin{array}{c}\text { Horas } \\
\text { según } \\
\text { norma }\end{array}$} & \multicolumn{2}{|c|}{$\begin{array}{c}\text { Moretag } \\
\text { adaptado }\end{array}$} \\
\cline { 3 - 4 } & Horas & Desv. \\
\hline & 43.118 & & \\
\hline 1,402 & 31.405 & 33.234 & $5,8 \%$ \\
\hline
\end{tabular}

\begin{tabular}{|l|l|l|l|}
\hline 1,402 & 31.405 & 33.234 & $5,8 \%$ \\
\hline 1,534 & 39.073 & 41.451 & $6,1 \%$ \\
\hline
\end{tabular}

\begin{tabular}{|l|l|l|l|}
\hline 1,202 & 31.123 & 33.141 & $6,5 \%$ \\
\hline
\end{tabular}

\begin{tabular}{|l|l|l|l|}
\hline 1,522 & 32.722 & 33.021 & $0,9 \%$ \\
\hline
\end{tabular}

\begin{tabular}{|l|l|l|l|}
\hline & 36.019 & 37.725 & $4,7 \%$ \\
\hline
\end{tabular}

\begin{tabular}{|l|l|l|l|}
\hline 1,534 & 35.715 & 37.889 & $6,1 \%$ \\
\hline & 16.633 & 16.626 & $0,0 \%$ \\
\hline
\end{tabular}

\begin{tabular}{|l|l|l|l|}
\hline & 16.633 & 16.626 & $0,0 \%$ \\
\hline & 33.905 & 33.909 & $0,0 \%$ \\
\hline
\end{tabular}

\begin{tabular}{|l|l|l|l|}
\hline & 33.905 & 33.909 & $0,0 \%$ \\
\hline 1,055 & 36.087 & 40.292 & $11,7 \%$ \\
\hline
\end{tabular}

\begin{tabular}{|l|l|l|l|}
\hline 2,000 & 24.013 & 24.437 & $1,8 \%$ \\
\hline
\end{tabular}

\begin{tabular}{|l|l|l|l|}
\hline 1,322 & 33.491 & 36.229 & $8,2 \%$ \\
\hline
\end{tabular}

\begin{tabular}{|l|l|l|l|}
\hline 1,962 & 17.583 & 17.492 & $-0,5 \%$ \\
\hline
\end{tabular}

\begin{tabular}{|l|l|l|l|}
1,503 & 46.430 & 49.480 & $6,6 \%$ \\
\hline
\end{tabular}

\begin{tabular}{|l|l|l|}
16.936 & 16.936 & $0,0 \%$ \\
\hline
\end{tabular}

\begin{tabular}{|l|l|l|l|}
\hline 1,268 & 32.062 & 34.834 & $8,6 \%$ \\
\hline
\end{tabular}

\begin{tabular}{|l|l|l|l|}
\hline 2,005 & 31.258 & 32.332 & $3,4 \%$ \\
\hline
\end{tabular}

\begin{tabular}{l|l|l|l|}
2,675 & 27.143 & 27.390 & $0,9 \%$ \\
\hline
\end{tabular}

\begin{tabular}{|l|l|l|l|}
\hline 5,903 & 34.751 & 32.523 & $-6,4 \%$ \\
\hline
\end{tabular}

\begin{tabular}{|c|c|c|}
\hline 0 & I & II/III \\
\hline 0 & 20 & 80 \\
\hline
\end{tabular}

\begin{tabular}{|c|c|c|c|}
\hline \multirow{2}{*}{ IPD } & \multirow{2}{*}{$\begin{array}{c}\text { Horas } \\
\text { según }\end{array}$} & \multicolumn{2}{|c|}{$\begin{array}{c}\text { Moretag } \\
\text { adaptado }\end{array}$} \\
\cline { 3 - 4 } & norma & Horas & Desv. \\
\hline & 61.101 & & \\
\hline 1,600 & 35.840 & 38.129 & $6,4 \%$ \\
\hline 1,798 & 45.696 & 48.772 & $6,7 \%$ \\
\hline 1,360 & 35.912 & 38.529 & $7,3 \%$ \\
\hline 2,083 & 44.800 & 45.314 & $1,1 \%$ \\
\hline 1,000 & 53.760 & 56.307 & $4,7 \%$ \\
\hline 1,798 & 41.769 & 44.580 & $6,7 \%$ \\
\hline & 39.137 & 39.120 & $0,0 \%$ \\
\hline 1,114 & 53.330 & 53.300 & $-0,1 \%$ \\
\hline 1,082 & 36.915 & 41.313 & $11,9 \%$ \\
\hline 2,493 & 29.926 & 30.513 & $2,0 \%$ \\
\hline 1,480 & 37.453 & 40.771 & $8,9 \%$ \\
\hline 2,688 & 24.084 & 23.933 & $-0,6 \%$ \\
\hline 1,750 & 53.990 & 57.886 & $7,2 \%$ \\
\hline & 39.850 & 39.850 & $0,0 \%$ \\
\hline 1,400 & 35.363 & 38.639 & $9,3 \%$ \\
\hline 2,500 & 38.886 & 40.403 & $3,9 \%$ \\
\hline 3,500 & 35.482 & 35.845 & $1,0 \%$ \\
\hline 8,885 & 52.307 & 48.561 & $-7,2 \%$ \\
\hline & & & \\
\hline
\end{tabular}

Normativas en las que Moretag propone mayor número de horas para el mix $\longrightarrow 14$ Normativas en las que Moretag propone menor número de horas para el mix $\longrightarrow 2$ Normativas en las que Moretag no afecta al número de horas para el mix $\longrightarrow 2$

$\left.{ }^{\star}\right)$ Normativas en las que el factor $p$ no tiene incidencia porque únicamente contemplan ratios de grados II/III.

Fuente: Elaboración propia. 
Distribución de plazas por grados de dependencia

\begin{tabular}{|c|c|c|c|}
\hline 0 & I & II/III & Total \\
\hline 0 & 20 & 80 & 100 \\
\hline
\end{tabular}

Extremadura

Castilla-La Mancha

$-148$

Navarra

$\begin{array}{r}\text { Madrid } \\ \hline \text { Andalucía }\end{array}$

Aragón

Castilla y León

Cataluña

Murcia

Canarias*

La Rioja*

Bizkaia

Asturias

Araba

Valencia

Cantabria

Baleares

Galicia

Gipuzkoa

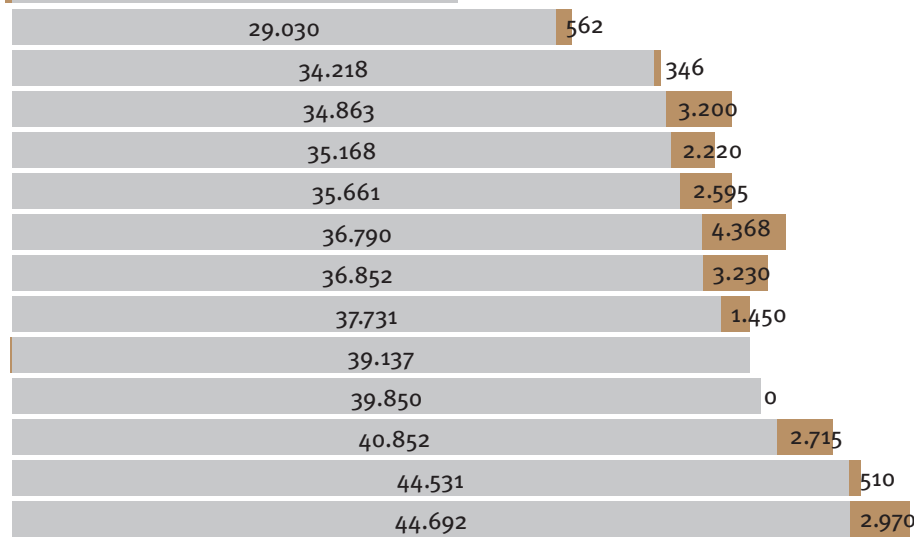

\begin{tabular}{|r|c|c|}
\hline-3.621 & 50.443 & \\
\hline-33 & 51.036 & 2.419 \\
\hline & 51.072 & 3.767 \\
\hline
\end{tabular}

60.385

Horas normativa

Complemento hasta MORETAG adaptado

* Las normativas de estas comunidades solo contemplan ratios de grados II/III, y en consecuencia, el número total de horas se corresponde con 80 plazas de grados II/III.

Fuente: Elaboración propia.

es más” adquiera dimensión de realidad, quizá con más frecuencia de la esperada. A pesar de ello, abogaremos por plantillas de personal capaces, por un lado, de dar respuesta a las necesidades de cada mix de dependencia y, por otro, coherentes con un patrón teórico de comparación estatal.

A diferencia de la divergencia intercomunitaria, el panorama estatal descrito por la adaptación de Moretag converge hacia la necesidad de incrementar las ratios, en mayor o menor medida, para tres de cada cuatro normativas analizadas. En términos generales, podría afirmarse que la concordancia no es algo distintivo del sector si lo analizamos desde la perspectiva que ofrece Moretag. No obstante, las desviaciones observadas no son especialmente insalvables si atendemos a los resultados de la Tabla 8. Es cierto que algunas normativas podrían estar más descompensadas que otras respecto de la supuesta trazabilidad de Moretag (casos de Castilla y León, en sentido negativo o de menor ratio que la propuesta por el factor $p$, y de la Comunidad Valenciana en el positivo o de mayor ratio), pero en ningún caso la diferencia es tal como para considerarla absolutamente inabordable desde los correspondientes órganos de gobierno. Los distintos observatorios, de ámbito autonómico o estatal, deberían transcender determinados enfoques limitativos y cuestionarse si la atención residencial a personas mayores con dependencia es realmente un derecho subjetivo y, como tal, debe estar sujeto a una misma interpretación para toda la ciudadanía de una misma unidad político administrativa (diputación foral, comunidad autónoma o Estado).

\section{Conclusiones}

De este análisis podemos extraer una serie de conclusiones:

- La producción de servicios residenciales para personas mayores no es sino la combinación de diferentes inputs - catálogo de tareas-a distintas intensidades en función de una tipología de usuario que requerirá de la cobertura de un conjunto de necesidades.

- El índice Barthel, dada su implantación en la mayoría de las organizaciones del sector por ser parte integrante de softwares generalistas, produce tal nivel de sinergia que no debe ser obviado. Desde el simple cumplimiento de las valoraciones Barthel programadas se abre un horizonte de gestión de datos que, a su vez, podría derivar en el rediseño recurrente del método para hacerlo progresivamente más eficiente. 
- Cada usuario presenta diferentes intensidades de gravedad -ayuda de tercera persona- para cada módulo del índice Barthel-CIF, lo que sugiere, probabilísticamente, la coexistencia de infinidad de perfiles. La inoperatividad de este hecho para la gestión eficaz de una red pública supone el arbitrio de un sistema alternativo que solvente los problemas operativos. Surge así la idea de isogrupo, o grupo de isodependencia, como perfil medio que aglutina y representa a un grupo homogéneo de diferentes perfiles Barthel-CIF. Estos isogrupos, además, deberían estar ineludiblemente vinculados a los grados de dependencia del BVD.

- El método no persigue encasillar a las personas mayores usuarias de servicios residenciales (entendido el término encasillar como "clasificar personas con criterios poco flexibles", según acepción de la Real Academia de la Lengua). Si algo ambiciona el modelo es el conocimiento de la composición combinada de módulos (AVD del Barthel-CIF) y niveles de dependencia para la identificación de perfiles de atención. Consecuentemente, se reivindica la redistribución de recursos de atención directa conforme a las necesidades reales de cada centro, fueran las mismas medidas conforme al índice Case-Mix o al número de residentes pertenecientes a cada isogrupo definido.

- Moretag es una referencia contextualizada en un escenario sociopolítico concreto. Moretag adaptado propone la proyección homogénea del modelo a otros contextos. Un panorama tan disperso como el que presenta el Estado en materia normativa induce a la definición cualitativa y cuantitativa de un factor de equivalencia entre el contexto original y otros alternativos. La adaptación no solo es necesaria cuando pretendemos dotar de universalidad al método; es incluso imprescindible cuando queramos dotar de verosimilitud a la herramienta para que el contraste sea fidedigno.

- El modelo desarrollado no se plantea como solución para resolver el problema de la dispersión estatal, responsabilidad que únicamente compete a los diferentes órganos de gobierno, últimos responsables para dinamizar voluntades políticas que reduzcan la divergencia existente en un anhelo por cumplir con el principio de solidaridad interterritorial (Gómez, 2018).

- Moretag combina práctica -más de dos décadas de ajuste de plantillas de personal para responder de las necesidades reales de atención contrapuestas a las exigencias del Decreto 41/1998 del Gobierno Vasco-y ciencia para correlacionar tiempos de gerocultor/a y nivel de dependencia en un determinado contexto sociopolítico. Inferir de esta experiencia razones suficientes para que se constituya en una suerte de oráculo estatal sería una osadía imperdonable. Sin embargo, aceptada la transversalidad del catálogo de tareas directa e indirectas propuesto por Moretag, el contraste teórico del modelo con los condicionamientos propios de cada normativa (ratios y jornada anual) es un ejercicio que permite objetivar un mapa estatal de potencialidades autonómicas desde una referencia común. En sí misma, esta posibilidad se vislumbra como un incuestionable avance hacia la convergencia dentro una misma unidad político-administrativa.

- El enfoque no se opone ni dificulta la continuidad del know-how de cada centro. En la medida en que tan solo aspira a ser una referencia para el dimensionamiento de plantillas de atención directa, en absoluto busca conflicto alguno con las culturas organizativas de cada centro residencial ni con sus sistemas de gestión. Se limita a proponer lo que se necesita para que cada organización lo amolde a su filosofía de trabajo. 
AUREN (2019): DENAdat, una herramienta para el dimensionamiento de plantillas de auxiliares de geriatría, < https://www.auren.com/es-ES/ contacto/1109/bilbao .

CALVO, F. (1990): Estadística aplicada, Ediciones Deusto.

CÁMARA, J. (2019): "Las previsiones de evolución del sector para 2019-2020 apuntan a una prolongación del crecimiento del volumen de negocio", Dependencia.info, 11/10/2019, < https:// dependencia.info/noticia/2931/actualidad/ las-previsiones-de-evolucion-del-sector-para2019-2020-apuntan-a-una-prolongacion-delcrecimiento-del-volumen-de-negocio.html〉.

DÍAZ DÍAZ, B. (2012): "Estimación del coste de la atención a la dependencia en centros residenciales y de atención diurna o nocturna", Zerbitzuan, 52, SIIS Centro de Documentación y Estudios.

GÓMEZ ORDOKI, A. (2014): "Valoración de la carga de trabajo de auxiliar en residencias para personas mayores (1 1 - parte: modelo teórico), Zerbitzuan, 57, SIIS Centro de Documentación y Estudios.

- (2018): "Precios, ratios y costes en la prestación de servicios residenciales para personas mayores: análisis comparativo entre Gipuzkoa y el resto del Estado", Zerbitzuan, 65, SIIS Centro de Documentación y Estudios.

IMSERSO (2011): Modelo de calidad de vida aplicado a la atención residencial de personas con necesidades complejas de apoyo, Imserso, 〈https://infoautismo.usal.es/wp-content/ uploads/2015/10/04._Libro.pdf〉.

KNAPP, M. (1988): La economía de los servicios sociales, Escola Universitària de Treball Social, La Llar del Libre.

LARES EUSKADI (2018): MORETAG: Modelo referencial de asignación de tiempos de auxiliar de geriatría (www.moretag.org), 〈http://www.lareseuskadi. org/uploads/media/MORETAG-Lares_Euskadi. pdf>.

MARTÍNEZ RODRIGUEZ, T. (2016): “Auxiliares y gerocultores/ as, profesionales clave en la ACP”, <http:// acpgerontologia.blogspot.com/2016/11/ auxiliares-y-gerocultoresas.htmls.

MOLINA SCHMID, A. (2010): Los requisitos de acreditación de residencias para personas mayores. Normativas autonómicas sobre ratios y formación mínima del personal para residencias privadas para personas mayores, Lares Federación. 